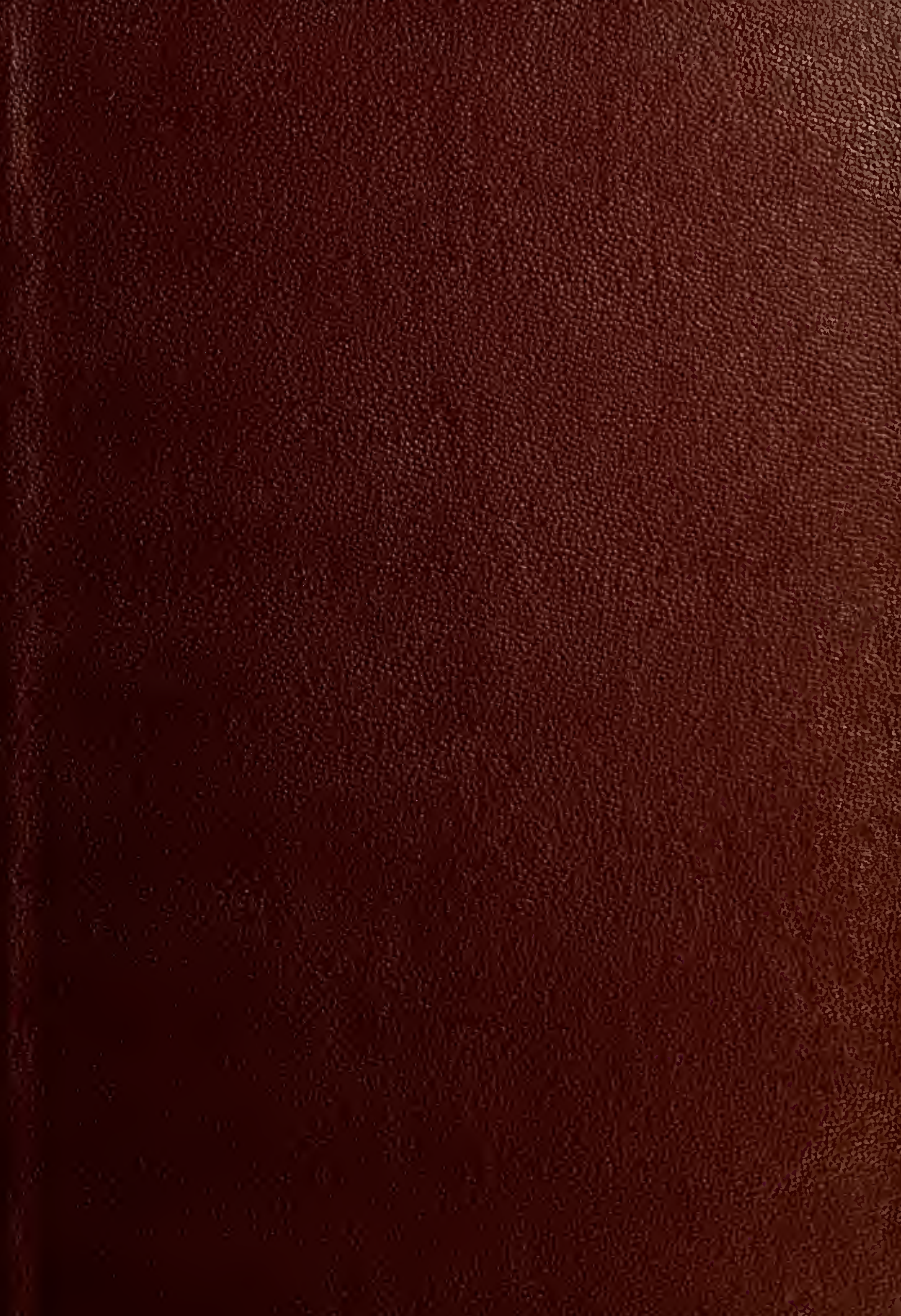




\section{HARVARD UNIVERSITY}

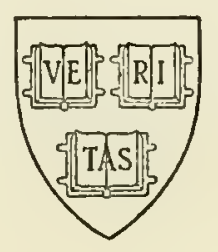

\section{LIBRARY}

OF THE

Museum of Comparative Zoology 




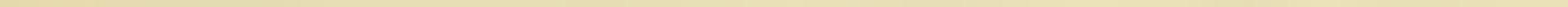



D. Stemstra-Jousaint 1835 MUSEUM OF COMPARATIVE ZOOLOGY PAMPHLET COLLECTION

tram urinam secernintion am, Selah the.

$$
\text { row } 11
$$

MUS. COMP. ZOOL

LIBRARY.

SER $15 \lg 64$

HARVARD

UNIVERSITY. 

A. J. D. STEENSTRA TOUSSAINT, EX PAGO ALPIIEN,

MEDIGINAE CANDIDATI IN ACADEMIA GRONINGANA,

\section{$\mathrm{R} \mathbb{E} \& \mathrm{PON}$ S I O}

A D

QUAESTIONEN ZOOLOGICAM, A NOBILISSIMO ORDINE DOGTRINARUM PHYSICARUM ET MATIEMATICARUM IN ACA-

DEMIA LUGDUNO-BATAVA A. MDccexxxIV PROPOSITAM:

"Quacritur descriptio anatomica organorum urinam secernentium in piscibus "et comparalio physiologica cum iisdem partibus in reliquis animalibus."

QUAE PRAEMIUM REPORTAVIT

D. IX MENSIS EEBRUARII A. MDGGGXXXv. 



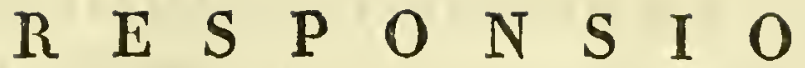

\section{A D \\ QUAESTIONEM Z OÖLOGICAM.}

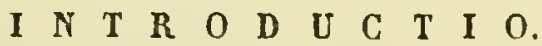

A natome comparata, quin summum praestel Physiologiae emolumentum, nemo unquam duhitavit. Hlinc recte Buffon: "s'il n'existaicnt, inquit, point d'animaux, la na. "ture de l'homme seroit éncore plus incomprélhensible." Et II aller: " anatome bruto. "rum plus boni fecit in plysiologia, quam anatome corporis humani. Situm, figuram. "magnitudinem partium ex homine disci praestat; utilitates cl motus partium animalibus "fere debemus."

Quod jam probatur ratione, qua plurimae funclioncs nostra imprimis actate majore in luce collocatae sunt. Quid enim certum haberemus de digestione, nisi multa experimenta in animalibus facta essent; quid de bilis secretione, nisi hepar caeterorum omnium animalium exploratum esset cognitumque, uisi etiam ex anatome comparata ratio esset intellecta, quae in diversis animalibus inter pulmones el jecur interceclit. Quod cum verum sit, merito suspicari licet fore, ut cognilis omnibus omninm animalium organis, eae quoque physiologiae partes magis explicentur, quam luc usque fieri contigerit.

Secretio urinae, cum nondum sit adeo explicata, ac reliquac multae functiones, cum. que organa urinam secemenlia ipsa apud omnia animalia nondum ita sint explorata, ut illa physiologine pars jam satis inde elucidari poluerit, non miramur nobilissimae placuisse facultati in certamine literario proponere quaestionem, quae tum in exponenda versetur fabrica systematis uropoëtici in piscibus, tum in comparanda hac fabrica cum ea ejusclem systematis ex animalibus reliquis.

Jam vero milii hanc quaestionem legenti valde quidern objectum plactit, idque adeo, ut opus eliam aggredirer, sed mox virium tenuitas cum magna ipsius rei difficultate, piscium simul satis magna copia deficienti, itaque omnia mili suaserunt discedere a labore incepto. Neque nunc vobis, viri Clarissimi, proponere potuissem hanc qualemcumque responsionem, nisi in meum solum commodum absque ulla jam praemii spe in incepto 
labore perrexissem. Attamen quo diutius evolverem, propria nanu faclas icones cum rem ijsam tractarem, pisces explorarem, scriptores iis aliorum virorum compararem, eo magis quoque dificultas rei sensim mili aliquomodo minor ficri videbatur; atque nune accijuenti mili novos pisces, novosque libros, consilium rediit, ut vires in elaboranda 'juaestione prericlitarer. Ita igitur natum est, quod hic jam prodit periculum, quodque ut cum venia atıue tota, quac Vobis est, humanitate accipiatis oro atque oro.

Secuentem in tractatu ordinen secutus sum:

pars prima continet auatomicain descriptionem organorum urinam secernentium in piscibus.

l'arte secunda exponitur comparatio physiologica liarum fartium cum iisden reliquorum animalium.

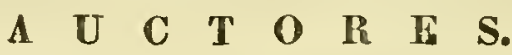

No. 1. Ger. Blasius, Anatome Animalium, Amstel. 1681.

« 2. H. Kull, beiträge zur Zoologie und vergleichenden Anatomic, Zweite AL. theilung.

4. A. Nlonro, Vergleichung des Baues und der Physiologie der Fische mit dem Bane des Ilensclien und der übrigen Thiere. Uebersetzt v. J. G. Schneider, Leipzig 1787.

"4. G. Guvier et Valeneiennes, Ilistoire naturelle des Poissons, Paris IS2S.

"5. G. Guvier, Vorlesungen üjer vergleichende Anatonic. Uebersetzt von J. F. Meckel, Lcipzig 1810.

"6. L. Huschke, Ueber die Karotidendrüsen einigger Amplibien. In des Zeitschrift für Physiologie von Tiedemann und Treviranus, Tom. 1V. pag. 116.

"7. In agendie et Desmoulins, Ueher die Anatomic der Lamprete. Im Deutschen Archire für die Physiologic von J. F. Meckel, Tom. VII. p. Ig. 236.

" 8. J. Davy, Ueber die Harnwerkzeuge zweier Froscharten. Im Deulschen Arehive vou J. F. Heckel, Tom. ViII. prag. 334.

" 9. M. IV. Plagge, Ueber die Urinsecretion der Blase. Im Arehive für Phys. von Meckel, Tom. VII. pag. $4: 9$.

" 10. J. F. Meckel, Ucber die Gallen und Harnorgane der Insecten. In dessen

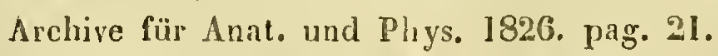

ה 11. J. Bleuland, Descriptio Musci Anatomici Academiae Rlıeno-Trajectinae.

"12. A. A. Berthold, Lelubuch der Physiologie. 
No. 13. A. R ömer, Handbueh der Anat. des Menschlich. Körpers.

" 14. L. Ja cobson, Système veneux particulier aux reptiles. In Oken's Isis. anno 1823. heft 12. pag. 1410 .

" I5. J. B Wilbrand, Ueber das Urinsystem der Insecten und Mollusken. In Oken's Isis anno 1321. Litt. Anzeig. heft 5. pag, 265.

" 16. Bloch, llistodre naturelle des poissons par Reué-Richard Castel.

"17. L. I', von Froriep's notizen No, S38.

"18. A ristoteles, de Animalibus, edidit Schneider.

" 19. 11. E. Hone, Descriptio Mnatomica Squali maximi (Lin). Journal de Physir. de Chimic, l'llistoire Natur. et des Arts par J.C. Delametherie, Juillet 1810. Ton. LXXI. pag. 24 j̃.

" 20. Natuurlylic listoric, of uitvoerige beschryving der dieren, planten en mineraleı, volgens het zamenstel van den lieer Linnaeus, 1ste Decl. 7de stuk, Amst. 1764.

«

(

《

21. C. G. Carus, Lehrbuch der vergleichenden Zootomic, Leipzig 1831. Tom. II. mit kujuforn.

22. H. Ra thke, Abhandlungen zur Bildungs. und Eutwickelıngs Geschichte des Menschen und der Thierc, zwei Theile, Leipzig 1832. mit kupfern.

23. H. Rath ke, Bemerkungen über den inneren Bau der Pricke oder des Petromyzon Fluviatilis des Linnaeus, Dantzig 1 S25.

24. 11. Ratikc, Beiträge zur Geschichte der Thierwelt, Abth. 4, mit 3 kupfern, Ilalle 1827.

25. H. Rathke, Ueber den Darmikanal und dic Zeugungsorgane der Fische, mit 5 Tafeln, Halle 1824.

26. A. A. Retzius, Observationes in Anatomiam Cliondropterygiormm, praccipue Squali et Rajac generun, Lundac 1819.

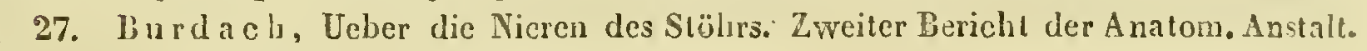
zu Königsberg, Leipzig 1819.

n. G. Guvier, liègne Animal. edit. 1829.

2!). Nilsson, Prodromus Ichthyologiae Scandinavicac, Lundac 1832.

31). L. J acobson, Ueber cin in sehr vielen Thicen vorkommendes besonderes Venensystem. In L. F. v. Frorieps Notizen $N^{\circ} .11$.

31. L. Ja cobson, Ueber eime wichtige Function der Venen. In Meckels Arehiv. für d. Physiologic, Band 3. pag. 147.

32. IVürzer, Chemische Untersuchung des Stoffes, welcher sich in den sogemanuten Gallengefässen des Schmetterlings der Seidenraupe (Phal. Bombyc. Mori) beliguct. In Meckels Archiv. f. d. Physiologie, Band 4. pag. 213. 
No. 33. Emmert und Hochstetter, Abhandlung üler die Eyer der Eidechsen. Im Archive f. Physiologie ron J. C. Reil, Band 10.

" 34. Jacobson et de Blainville sur l'existence des Reins dans les animaux mollusques. Dans le journal de Physique etc. par Ducrotay de Blainville, anno 1820. Tom. XCI. pag. 318.

* 35. Ferrein, sur la structure des viscères nommés glanduleux el particulièrement sur celles des reins et du foic. Histoire de l'Academie royale des seiences de Paris, 1749. pag. 439.

46. K. ron Schreibers, Ueber den Harn der Eidechsen. In L. W. Gilbert Annal. der Plyysik. Band 43. pag. 83.

* 37. I. U. H. Nicolai, Disquisitiones circa quorundam animalium venas abrlominales, praecipue renales, Beroliui 1823.

" 38. Jol. Nuller, Bildungsgeschichte der Genitalien, Düsseldorf 1830.

* 39. Jacobson, Dic Okenschen Körper oder die Primordial Nieren der Säugethie. re, Koppenhagen 1830.

* 40. Brugnalelli, Giornale di Fisica 1815.

* 41. Herold, Entwickelungs Geschichie der Selımetterlinge.

« 42. Rengger, Physiologische Untersuchungen über die thierische Iraushaltung der Iusecten, Tübingen 1817.

« 43. Fink, Pracside J. F. Heckel de Amphibiorum systemate uropoetico, Halae 1717.

"44. Journal de Pharmacic Tom. Vil. pag. 381.

“ 45. Fr. Tiedema n Pliysiologie des Menschen, Darmstad 1830.

4 46. Composition des $\Lambda$ pparcils génitaux, urinaires el intestinaux à lents points de rencontre dans l'Autruclıc et le Casuar, Mémoire du Muséum d'histoire naturelle, Tom. IX.

* 47. Memoires pour servir a l'histoire naturelle des animaux. 


\section{P A R S I.}

\section{DESCRIPTIO ANATOMICA ORGANORUM URINAM SECERNENTIUM IN PISCIBUS.}

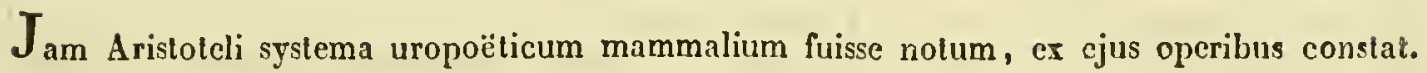
De avium vero, piscium et reliquorum animalium organis urinam secernentibus nullam habuit scicntiam. Elenim : "Renes autem, inquit, et vesicam quadrupedes omnes ha"bent, quae scilicet animal pariunt. At oviparorum nulla, neque enim aut avis aut " piscis." (1S. Tom. II. Hist. 2. Gap. 12.)

Interim systema illud etiam in animalibus inferioris ordinis necessario jam adesse, facile credit quicuncine ad insignem magnitudinen et summum perfectionis gradum attendat, quem illa organa in mammalibus ostendunt. Utque enim liomo non tamquam infans plures annos natus in lucem editur, sed potius inobservabili modo ex niliilo fere ad exsistentiam transil, sic etiam systema uropoëticum in regno animali sensim majorem perfectionis gradum attingens, non uno prodire potest tam magnum tam perfectum.

Jam vero nostris temporibus nemo est qui dubitet de hujus systematis praesentia in piscibus, nec etian in animalibus quae evolutionis minorem gradum attingant; nam etiam in piscibus illud systema tam perfectum cernitur, ut jam in inferioribus animalibus hoc systema adsit sit necesse.

Systema uropoëlicum piscium in univcrsum pariter ac in homine constat ex organo urinam secernente, ex alio deferente, ex tertio quod cam colligit et denique quod urinam ex corpora foras educit; scilicel e renibus, urcteribus, vesicà et urethra, quorum trium ergo officium versatur in climinando humore nulli corporis functioni amplius inserviente.

\section{R E N S.}

Hoc organon primarium systematis uropoëtici in piscibus duples cernitur, esceptis Squalis, si Retzï observationibus fides habenda est. In utroque latere columnae vertebralis collocatum est supra vel juxta partes genitales; neque uli in mammalibus ren dexter et sinister semper omnino a se invicem separati inveniuntur. Nam lieet renes in Rajis quidem in utroque latere spinae sint collocati, ut nulla ratione, nisi mediatim per 
A. J." D, STEENSTRA TOUSSAINT,

vasa et vesicam cohaereant, id tamen non in omnibus cacteris ila est, nam e contrario plurimi renes in posteriore parte ita in unam massam coalescentes habent, ut fere unum ren adèsse crederemus duobus lobis instructum; ita v. c. renes cernuntur in genere $\boldsymbol{P}$ erca, Trigla, Pleuronectile, Collo, et ex genere Raja in Raja Clavata. (Num. 21. pag. 652.)

Silus renum ut jam memoravi ad colımnam vertebrarum est. In aliis usque ad capnt adscendunt, totum cavum abdominale percurrentes (Cotius Scorpio, Symbranchus immaculatus, Anarrkichas Lupus (Num. 2. pag. 171.) Collus Calaphractus, (ibid. pag. 179 )

In aliis demum piscibus pro varia corum magnitndine et inprimis longitudine majorem ninoremre parlem columnae spinalis occupant (P'ztromyzontes, Squalus Acanthics (ilbid. pag. löl.) Trachinus Draco (ibid. pag. 175.) Salmo. (Num. 21. pag.65l. S. 726.)

Renes ergo diversa ratione sunt locati, imo in mo eodemque genere non semper aequali modo, cujus rei Pleuronectes Solea sit exemplo. In hoc enim pisce pars inferior quae simul posterior est in cavitate quadam extra abtomen, qua in ceteris hujus generis ovarium sinistrum conlinetur, est dispositum. Posilio renum relaliva ea est, ut in piscibus quibus vesica natatoria sil, supra eam sint collocuti, et hace igilur vesica multun conferat ad renes in loco quem occupant retineudos. (Gadus, Trigla, Cyprinus.) In universum supra omnia intestina suam sedem habenl et ureteres descendendo ad vesicam perveniunl, ut etiam urethra pone anum. Oraria in utroque latere infra vel juxta renes collocata, conspiciuntur. Renes in loco quem oceupani relinentur peritoneo, quod totum cavum abdominale tegens etiam renum faciem inferiorem tegit. In is piscibus, qui vesica natatoria sunt instrueti hace renes tegit, et in utroque latere costis fortibus ligamentis adbaerens impedit, quominus ex loco moveri possint. - Si haec vesica natatoria dést, renes obducuntur membrana quadam propria, fortiori, quae unice huic scopo inservire videtur. Naturae est fibrosae fortis et in utroque latere fortibus ligamentis tendinosis costis adfixa. Perspicue cernitur liaec membrana in Pleuronectile et Perea. Vasa denique eliam, quae renes intrant multum conferunt, ut in loco remaneant.

In Petromyoonte fuvzatili renes a posteriore tertia parte abdominis ad lujus cavitatis finem fere decurrunt, et quidem oblipue a latere interno el superiore, versus exteriora et inferiora. Latus exterius musculos abdoninales altingit, margo autem superior subrotundus sinni adhaeret co fere loco, quo iste sinus utriusque lateris posteriorem cavam venain tegit (No. 23. pag. 51.)

Forma renum valde differt, licet rulgo reperiantur longi angustique. In Lophio $P_{i s-}$ catorio brevissimi sunt, ovales, magnitudine ovo gallinaceo aequales. (No 2. pag. 180.) 
In universum si renes ad caput usque adscendunt, vulgo crassiores ibi fint el vario modo lobati observantur; si vero non tam longe se extendunt, tunc ut plurimum in apicem excurrunt. In Raja Rubo ubique ejusdem fere sunt latitudinis, uti ex Tab. I. Fig. 4. patet, ubi naturali magnitudine sunt delineati.

De Raja clavata L., ren juquit Retzius (No. 26. pag. 11.) soleae equi similis, alis antrorsum versis; in Raja fullonica (ibid. pag. 16.) renes sunt disjuneti semilunares; in Raja Batide (ihid. pag. 21.) renes fullonicae multum dissimiles ad clcvatae figuram magis accedunt: Duo crim adsunt graciles, oblongi. In Squalo Glauco (ibid. pag. 8.) ren naviculac fere formam habet; posterius nempe et superne carinae in modum compressus, anterius tenuior, sed depressus. Latus inferius medium sulcum ureteres foventem hahet, cujus margines transverse fissae sunt, quod loborum locum significat. Inpressionibus costarum in avium pulmonibus haud dissimiles sunt. In Squalo Acanthia renem frre ut in Squalo Glauco esse idem testatur (ibid. pag. 11.). Ergo, pergit Doctissimus Iefzius, in illis dnobus Squalis ren formam habel cunei longi, ad posteriora rotunde compressus et anterius depressus. $\Lambda$ pud Rajam clazatam solummodo uterque ren in unmm concretus est, in fullonica et Butide aperte divisus. Additae icones hanc figuram recte explicant.

- Renes Petromyzontis fluviatilis (No. 23. pag. 50.) quos Home pro testiculis habrit, longi sunt et fere ubique ejusdem latitudinis et crassitiei; antrorsum corum fines subrotundi sumt; videtur quidem corum arterior pars a multo angustiore et fili instar teaui parte incipere, verum haec pars continuatio renis habenda non est, sed adiposa natura vel maxime a rene differt.

De hocce corpore adiposo aliam protulit sententiam Doct. Ret:ius: postquam enim descripserit hoc corpus in diversis piscibus, in Squalo Glauco et Acanthia, in Raja Batide caet. de Raja clavata L. (No. 26 pag. 11.): "Substantia illa, inquit, pallida flavescens, quam in dorso renis apud Squalos memoravimns, merlullac nerveae similis, ureteribus paralela, et fere eadem crassitie. Quicunque ad propriam adipis speciem in hisce piscibus non attendit, illam panniculum aciposum renis haberet. Hembrana ei est perspicua bis constricta. Quam vero substantiam, quoniam hic magis ac in Squalis a rene scjuncta est, glandulam suprarenalem ese mihi indicio fuit." - Cum vero substantia haec memorita saepe etiam reperitur in latere externo renum, ut et in regione dorsali aliorum piscium, exempli causa Blennï vivipari et tam adspectu suo quam ceteris proprietatibus convenit cum adipe, magis equidem sententiam viri Clar. Rathlie amplecterer, qui naturam hujus corporis adjposam luculenter demonstravelit (No. 23 pag. 52. et No. 24. pag. 92 et 94.)

Renes Triglae llimundinis in posteriore parte ubi coaluesunt sat lati sunt, anteriora versus minorem habent massam et ad caput iterum merescunt. 
Cottus Scorpio renes habet in anteriore parte quodam capite triangulari instructos, quod per partem angustiorem cum cetero rene communicat. (Tab. I. fig b.) In Pleuronectide maximo lobati conspiciuntur, in Pleuronectide Solea singulari modo pars inferior posterius extra cavum abdominale se extendens, crassior est; in universum in hoc genere piscium renes eo loco mole augentur, quo a columna vertebrali inferius de. scendunt. - Quibus nunc paucis exemplis jam satis constat figuram renum in piscibus eandem semper non esse sed diversam; imo in uno codemque genere maguas observari diversitates. Sic etiam 1)oct. Fílel (No.2. pag. 156.) renes Ga!li Carbonarii describit crassos, sed breves, non ad spinam extensos sed colli vertebris appositos, licet aliae hujus generis species, Gadus Molva, Gadus Mustela, Gadus Aeglefinus, renes oblongos et ad spinam decurrentes habent. Cum nullus fere, qui mili ad manus est auctor ad magnitudinem et pondus renum attenderit, etiam ego ex paucis meis observationibus conclusionem generalem proponere vix audeo. Tenebant autem renes corum quos ego investigavi pisces, inter tertiam et quintam partem longitudinis totius piscis, et profecto videntur renes multo majores esse in piscibus, ratione habita lotius corporis, quam in reliquis animalibus, qua de re etiam consentiunt viri Clar. Cuvicr (No, 5. pag. 637.) Römer (No. 13. pag. 381.) Berthold? (No. 12. pag.577.) aliique.

In Squalo Glauco, cujus sceleton ab apice nasi ad apicem caudae sex pedes longum crat, renis longitudo acqualis crat quindecim digitorum, latitudo autem trium digitorum (No. 26. pag. S.) Color renum ex rubro in coerulcum, fuscum vel nigrum abit, etiam flavi reperiuntur uti Bloch memorat de Blennio Pholide (No. 16. Tom. II. pag. 4.) Consistentia in universum mollis, posteriora versus paululum indurescens. Anterius vero saepe spissitudine sanguinem paululun coagulatum non superant. Ita in genere Trigla, Gado, Perca, Cyprino, Pleuronectide el aliis.

In Accipensere substantia renum jam paulo durior dicitur, pariter ac in Cycloptero Lumpo, Collo Scorpione, et Blennio viviparo (No.23. pag.53.) Etiam Squali et Ra. gae renes compactiores dicuntur quam celerorum piscium (No. 26. pag. 27.) Exsiccatione renum substantia ad massam pulverulentam fusco-nigram reducitur.

Facies renum inferior tegitur peritonaeo et in nonnullis multa tela cellulosa quod inprimis in Trigla observavi. Renes ipsi circumdantur membrana Lenuissima propria, saepe coeruleo vel argenteo pigmento tincta, quae tam arcte cum substantia ipsa renum cohaeret ut difficilime ab ea solvi possit.

Adspectu exteruo renes universe homogeneam, indefissam ostendunt massam, multis vusis perreptatam, quod optime applaret in generibus Cyprino, Perca, Pleuronectide, Trigla, et in universum in omnibus minoribus piscibus. Nonnunquam renes ex minoribus partibus conflati apparent, licet hae partes non ita separatae sunt ac in nonnullis mammalibus, cujus rei exemplo sit Raja, cujus renem sinistrum fig. 4. tab. I. ad na-, 
Iuram delineavi. Etiam ex Tab. II. Fig. 4., quac est decima in opere A. Mronroi, a Schneidero edito, dilucicle patet renes ex pluribus partibus minoribus constare.

Substantia rerum interna plane est homogenea in pluribus piscibus, adeoque ut duae illac substantiae quas hominum v. c. renes ostendunt nullo modo discerni possint. In nonnullis vero generibus, quo pertinent Gadhis, Esox et Pleuronectes grumi vel parvi globuli nigri huic massae homogeneae inhaerent, qui si microscopii ope investigantur, ex innumeris etiam minoribus globulis constare apparent; plane autem cvanescunt illa corpuscula si substantia a!jua diluitur. Cum armato oculo contempleris renis frustulum id ex convolutis duclibus sanguiferis et uriniferis confectum apparet, quibus vero magis minusve aperte inhaerent illa dicta corpuscula. Omnium denique ductuum, et corpus. culorum intervalla materia homogenea, seri sanguinis simili, impleta sunt.

Retzius (26. pag. 8-9) renem Squali Glauci his verbis descripsit: Parenchyma obscure rubrum lineis lucidioribus fasciatum glomerulisque contextum est Fibrae tendineae parenchyma transeunt divergentes, maxima ex parte ab aponeurosi inferiore ortae. Tunica renis crassa valida et fibrosa, tendinum in modum splendens, aponeurosi dicta connexa. - Rajae clavatae renem (ibid. pag. 11.) dicit ex duodeviginti lobis constare interne connexis obscure carneis, propria membrana tenui et tenaci inclusis. Ureteres e quoque lobo ramum accipiunt. In Raja Batide lidem vir Doctiss. (pag. 21.) in quoque rene undecim sed parum perspicuos lobos vidlit. Denique in universum de structura renum Squali et Rajae disputans «vasorum, inqquit, uriniparorum radiculi in truncos penicillatim colligentur, ut trunci maximi uretercs sint. Parenchyna pallidius et minori vasorum sanguiferorum numero instructum est, quam in ceteris piscibus" (pag. 27.)

In Petromyzonte fluviatili renes uti Rathke testatur ( $\mathbb{N}_{0} .23$. p. 52.) constant ex tubulis parvis et penicillatim fere conjunctis, qui tela cellulosa inier se juucti, transversim per renes currunt versus ureteres inque eos aperti per multa foraminula ad exteriorem renis marginem locata.

De renibus Ammocoetis branchialis Rathke haec habet: "(No. 24. pag. 93.) Von der vorderen Hälfte dieses Kanales (ureter) gehen unter rechten Winkeln, und in kleinon Entfernungen von einander eine Menge äusferst zarter Gefässe ab, und wenden sich alle nach oben. Ein jọdes dieser Gefässe, von welchen die mittelsten am längsten sind, hat im Ganzen eine ziemlich beträchtliche Länge, und scheint ganz einfach zu sein. Ein jedes ferner ist zum grössten Theile knänelartig zusammen gewickelt, und seine Windungen werden nur durch eine sehr geringe Masse von Zell-Gewebe zusammen gehalten. Wenig Zcllgewebc auch hällt die einzelnen Kıäıel unter ein ander zusammen. Die ganze Ansammlung dieser Gefässknäuel, welche nichts anders, als die Harngefässe sind, befindet sich an dem untern Theile der vorderen Hälfle des Fettkörpers 
und hat wegen ihrer innigen Verbindung mit denselben das Ansehen, als wäre sie nur eine und-zwar die kleinere Abtheilung von-ilım. Um diesen Schein noch zu vergrösserı kommt hinzı, dass die Endstücke der besclıriebenen Gefüsse nicht mehr knäuelarlig zusammen gewickelt sind, und dicht bei einander liegen, sondern stark geschlängelt, und in mässigen Entfernungen von einander in den Fettkürper selbst eindringen, und innerlalb demselben von unten nach oben verlaufen."

In Petromyzonte Planeri renum structura media est inter eam illius visceris in $P_{e-}$ tromyzonte fluviatili et Ammocoete branchiali; nam primum ductus uriniferi quorum autem frequentia insignis est, minus penicillatim, quam in pelromyzonte fuviatili sed magis a se invicem separati in ureteres abeunt, et denique ijdem ductus etiam minus quam in Ammocoetc serpentino modo decurrunt. (No. 24. pag. 101.) In Squalo Miustela, vel potius in hujus speciei animalium embryone Ralhlie hanc renum structuran observavit. (No, 24. pag. 22.):

"Nachdem ich die Nieren," inquit, " mit ihren Harnleitern von der Rückenvand der Bauchlı̈hle abgelöset hatte, sah ich mil Hülfe des Microscopes, dass in ziemlich gleich grossen Entfernungen aus jedem Hamlejter eine Henge kurzer Gefässe hervorging, deren jedes sich bald nach seinen Urspunge in zwei oder mehrere verhältnissmässig ziemlich dicke sich allenthalben gleich blcibeade und cinfache (nicht weircr veristeltc) Gefässe zerspaltcte, welclie darauf nur durch sehr wenig Schleingewebe zusammen gelıalten, Ind dem :iusseren Ansehen nach sehr düunen Därmehen älnlich, eine ziemliche Arzahl durch ein ander gehende, Windungen machten, so dass ein jedes Pack sol̄cłer Gefässe in mehrerer Hinsicht einem in nalürlicher Lage befindlichen Dünndarme eines Sü̈gethieres ähnlich salı, Ein jedes durch einem eigenen Ausführungsgang mit dem Iarnleiter wie, sehon bemerkt in Verbindung slehendes Pack dieser Gefässe war von dem andern dureh einen den:lich wahmen!bnren und nur von weuigen Schleimgewebe ausgefülten Zwischenraum geschieden, und diese nur an $\mathrm{der}$ Rüetrenseite der Nierc deutlich wahrnelımbaren Zwisclıenrüume wurden an der Banchseite der Niere (wo sich das meiste Schleimgewebe befand, und von welcher aus man sich über die Beschaffenheit und den Verlauf der einzelnen Ifarngefässe nicht nähere Kenntuiss verschafea konnte) durch scichte Querfurchen angedeutet."

Systema vasornm animalium, quae non pulmonum ope sed branchiis spiritum ducunt, non tam multum difert' al, eodem systemate celerornm aninalium, quam id pro diversitatc apparatus respirationis exspectari posset. In mammalibus enim sangnis in subtilissimis vasorum pulmonalium ramulis ab acido earbonico liberatur et novo oxygenio restauratur; in piscibus autem respiratio perficitur in subtilissimis ramis insius anrlae, per branchia distributis. - Aorta enim a corde incipiens, arterias brancliales edit, quac a branchiis redemntes ad uaum magnum vas (aortam) confluunt, infra columnam 
vertebrarum suum iter absolvens. Statim post lianc suam formationem ao rta duos magonos ramos emittil ad intestina. Alias porro arterias in decursu suo infra columnam vertebrarum emiltit, quae supcriora versus circa corpora vertebrarum sese hectcutes allscendunt et in antcriore margine processuum spinosorum ad pinnas dorsales tendunt. Es his ramis adscendentibus iterum rami ad parictes abdominis dimittuntur. In substantiam renum ipsam minimae el paucae arteriae intrant, difficillime tantum mercurio adimplendac.

In Raja Batide, inquit Retsius (No, 26. p. 21.) renes sanguinem ex parte accipiunt ex arteriis, quae ex arteriis intercostalibus, mox ab aorta exurtis, originem capiunt.

In Petromyzonte fluviatili hanc arteriarum per renes distributionem Ruthke observavit (No. 23. p. 68.) In facie inferiore rel interna utriusque renis, justa cjus longitıdinem ct in medio rene arteria decurril satis magna, quae primo quidem adspectu ex aorta ipsa provenire vidctur, sed accuratius contemplanti manifeste apparel ad ejus certe fines aculos, nullo modo cum aorta conjuncta. Jam vcro si ex diligentia in illius arteriae originem inquiras, videbis magnam frequentiam parvasun artcriarum ad aequales distantias ex aorta sub angulis rectis ortarum, quae sinum abdominis venosum per:0rantes atque in duos vel tres minores ramulos fissae cum illa artcria in medio renc decurrenti se conjungunt hancque adco cum aorta uniunt. Interim alii quoque ramuli oriuntur ab utroque latere illius arteriae renis, penetrantque in cjus parenchyma.

Vasa venosa piscium certe si de renibus sermo est, multo majoris sunt momenti: Sanguis venosus e capite confluit in duo sat magna vasa (venae cavae antcriores) quae eundem habet decursum ac capitis arteriae et paululum pone cor se conjungunt cum duobus vasis e posteriore parte corporis oriundis (venae cavae posteriores). Quarum altera ortum e cauda ducens infra columnam vertebrarum decurrit ct per dextrum rencins ad cor viam prosequitur, ubi se conjungit cum rena cava dextra antcriore.

Altera vena cava posterior proveniens ex sinistro rene cujus sanguinem recipit, cum sinistra cava anteriore se conjungit. Plures deinde venae, quac $\mathrm{c}$ minorum venularum confluxu in parietibus abdominis formantur, ad renes adscendunt et in is iterum sese vario modo in minores ramos dividunt. Etiam ab ovariis, a resica urinaria, et e cauda majora vel minora vasa renes intrant diversa ratione per eos se distribuentia. Hancce dispositionem vasorum renalium in variis generibus reperi ex. gr. in generibus Gado, Perca, Cyprino (Tabula II. Fig. 3.) et aliis. Doct. II. Rathle similem vasorum dispositionem describit in Blennio viviparo. (No. 22. Tom. 2. p. 35.)

Multum autem abest, ut vasorum decursus apud omnes pisces descripta ratione sese habeat; in multis vasa alio modo distributa sunt, qund equidem praecipue in Trigla et Pleuronectibus observari. In his enim magna vena c cauda ad renem decurreus non 
aeque ac in $\boldsymbol{P}_{\text {ercâ }} \mathbf{v}$. c. renem percurrit ramọ minores recipiens, sed statim si renems intraverit se dividit in permultos ramos per totos renes distributos uno eodemque modo ac omnes ceterae venae ex diversis partibus corporis renes adeuntes. Omnia haec vasa, si in modum arteriarum subtilissimas ramificationes formaverint, eodem modo ac venae ad majores ramos sensim confluunt et per minora majorave ostia aperta sanguinem cffundunt in sinum quemdam magnum venosum. Hic sinus in inferiore facie renum a posteriore parte anterins decurrit et se in duos ramos dividit co loco quo renes a se invicem recedunt. Eodem modo ac venae cavac posteriores hujus sinus "utergue ramus se conjungit cum vena cava anteriore. Sinus mox descriptus e Trigla Tabula II. Fig. 1. est propositus, ubi incisione longitudinali aperlus est, ut ostia vasorum in conspectum venirent.

Expositis iis quae ipse viderim in diversis piscibus, jam paucis tradam, quae al aliis de renum venis sint observata, ut simul pateat non eadem semper ratione renarum ad renes pertinentium distributionem et decursum se habere, sed multiplici.

Vulgo, inquit Rathke (No. 25." p. 199.) omnes vel maxima certe venarum genitalium pars in Blennio viviparo et Ammodyle Tobiano in venas renales abit, et quidem cum pluribus ramis, pleuronectibus exceptis, in quibus cum vena cava conjunctae sunt, In Anmodyte Tobiano in sulco nempe superiore longitudinali partis genitalis simplicis, juxta longitudinem cadem ratione ac in Cyprino Carpo truncus venosus decurrit, sub angulis reclis omnes partis genitalis venulas recipiens. $\mathbf{A b}$ altero latere, idem venosus truncus quatuor quinqueve filamentorum instar tenues venulas ad aequales a se distantias emiltil, quae adscendendo circa dextrum renis marginem decurrentes in partem posteriorem venae renalis dextrae transeunt. In aliis piscibus, nempe in Blennio vivio paro, Esoce, Colti, Cycloptero Lumpo, Cobili fossili, in quibus vena longitudinalis desideratur, venulae ab utroque latere adscendentes ad plures ramulos confluunt, renes intrant, ut cum eorum venis se conjungant.

In IIarengis No. 25. p. 200.) in quibus genilales venae primum quidem eundem decursum tenent, ac in prioribus piscibus, truncus tamen e parte genitali dextra ortus ad latus sinistrum abit, el sese conjungit cum trunco venoso a parte genitali sinistra adscendenti. Qune vena hoc modo constitıla, posıquam sinistram partem renis perforavil, in renalem venam dextram transit, neque in hepar abit, uti credidit Kuhl.

In Petromyzonte fluviatili venae renales constituuntur permultis parvis venulis, quae ex renum substanlia exsurgentes parros truncos furmant, qui sanguinem suum non in venam cavam sed in sinum renosum abdominalem effundunt. (No. 23. p. 70.) Addit porro vir Clar. Rathke sanguinem venosum a musculis caudae, abdominis et a partibus

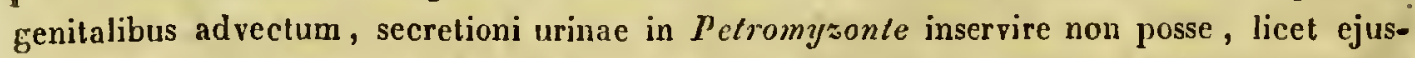
modi sanguinis venosi 1 sım apud alia animalia negare non vult (ibid. p. 72.). Ex iis 
quae dictae sunt, patet etiam renes in piscibus quadam specie systematis portarum esse insiructos. Jacobson (No. 14.) primus hoe renum portarum systema vidit idque non piscibus solum verum etiam avibus et amphibiis tribuit. Clar. Jacobson enim (No. 30.) sequenti modo loc singulare systema venosum describit: « in avibus, inquit, reptilibus et piscibus tres hujus systematis species observantur. In primce ex cute et musculis partis mediae corporis, parvi rami proveniuml, qui sese conjumgentes ab omni parte renes aggrediuntur et per eorum substantiam se distribuunt. - Secunda species ita efficitur, ut venae ex posteriore parte corporis venientes in hoc systema recipiantur. - Tertia species codem modo est formata ac secunda, hacce tantum differentia, quod a venis ex posteriore parte corporis, ramus dimittatur ad venam portac.

Jam vero quod attinet ad hoc singulare systema in singulis animalium classibus, in piscibus sub, unaquaque harum trium specierum nobis apparet. In plurimis enim piscibus in quibus hujus systematis primam speciem observanus, omnis sanguis cutis et musculorum a ramis venosis recipitur ad renes decurrentibus. Tali modo vasa decurrunt in Clupea, Cyprino et aliis. (Meis etiam observationibus hoc comprobatur. Antea enim jam locutus sum de tali dispositione vasorum in Gado, Perca et Cyprino, cujus ultimi renes cum vasis Tabula II.. Fig. 3. proposui.) Etian secunda species, pergit Clar. Jacolson, saepe in piscibus observatur. Omnis sanguis ex posteriore et media corporis parte ad renes ducitur; vena caudalis, statim si renes intraverit, duos majores ramos format, qui receptis caeteris venulis minoribus ex media parte corporis, per renum substantiam sese distribuunt. Quod ita vidimus in Raja, Esoce,. Squalo, Pleuronectibus caet. (Quod ad me attinct in Pleuronectibus et Trigla loc etiam observavi. In Esoce vero equidem non secundam sed primam lujus systematis speciem reperi.)

Tertia species a Clar. Jacobsonio in Muraena solummodo et in Lophio est reperta. Dimittit scilicet vena caudalis prater venas ad renes uuam ad venam portarum, ita ut sanguis posterioris et mediae corporis partis partim veniat ad renes partim ad jecur.

\section{UR TERES.}

Ureteres ubique sunt ubi renes reperiuntur, neque mirum cum urinam in renibus secretam foras educunt. Paucis exceptis (No. 17.) omnes pisces duos habent ureteres, aeque ac caetcra animalia quae duobus renibus sunt instructa. Ureteres duplici inprimis modo in renibus formantur, nempe vel ab anteriore parte renum oriuntur totosque renes percurrunt, vel a posteriore parte.

Formatio ureterum, si in anteriore parte renum locum habet, ita oblinet ut minimi ramuli ductuum uriniferorum in unum confluant et ab omni parte in toto decursu usque ad finem posteriorem renum sese inserant in ureteres, qui igitur majores et capaciores 
facti a renibus demum recedunt. - Hoe mox deseripto modo ureteres formalos vidi in Trigla Hirundine quorum delineatio Tabula I. Frgura 5. a reperitur; etiam in Cyprino ureteres totos renes percurrunt (Tabula II. Fig. 3 ). Exocoetus porro evolans, Petromyson fluviatilis Cotius Scorpio, Perca fluviatiiis, Esox et alii ureteres eadem ratione formatos habent ac dispositos.

In aliis piscibus in quibus ureteres non usque ad anteriorem renum partem prosequi possumus, ipsi eliam alio modo constituuntur. Etenim in illis ductus minimi uriniferi ab omni parte in posteriorem finem renum conveniunt el rete quasi formant ex quo ureteres proveniunt statim sat ampli et capaces. (Genus Gudus, Pleuronectes.)

Ureteres si a renibus decedunt, vulgo sibi appositi descendunt ut in Ciado, Tabula II. $F_{i g} .2 a, 2 b, 2 f$, vel separati usque ad eorum conjunctionem quod obtinet imprimis in is, qui vesica sunt destituti v. c. in Petromyzonte fuviatili et Cobili fossili. Ta. bula I. Fig. c. d. In universum 'per unum ostium commune ureteres urinam vel in vesicam vel foras emittunt; a qua regula unicam tantım legi exceptionem, nempe in $G \dot{a} s$. terosteo aculeato et Spinachia quorum quinque ad sex ureteres in 'iuoque latere per separata ostia urinam in vesican deducunt. Tabula II. Fig. 2e. (No. 17.). Eandem vero' dispositionem ipse observari in Raja clavata. Tabula.II. Fig. 5. Ureter enim ex anteriore parté renum urinam ducens, non per idem ostium ac caeteri ureteres vesicam intrat; ita ut in quoque latere duo adsint.

Ureteres si nullum adest obstaculum recta linea a renibus ad vesicam decurrunt, uti in Perca, Cyprino et alïs. In iis vero piscibus in quibus vesiea natatoria impedimento est, ureteres vel circa finem posteriorem liberum vesicac, se flectunt, rel si haec eo loeo nimis arcte adhaeret partibus ambeuntibus, inter vesicam et peritonaeum descendunt ad parietes abdominis. Rarius urcteres vesicam natatoriam perforant uti in Gado Pollaclico ( No. 17 ).

Fibrae longitudinales in majorum piscium ureteribus facile conspiciuntur (ibidem) et ex analogia concludimus eliam adesse in minoribus lieet ob subtilitatem oculo sese non offerunt. Interim ureteres Petromysontis flurialilis fibris carneis carere testatur Rathle (No. 23. pag. 53.).

- Capacitas et longitudo ejus partis ureterum, quae extra renes inter eos el vesicam posita est, variat pro genere et magnitudine piscium, sine ul'a proportione ad magnitudinem renum vel resieae, sic Gadus Callarias tres pedes longus vesicam habebat quatuor pollicum et dimidii; alter Callarias undecim pollices et dimidium longus vesica pollicis unius et dimidii erat instrıctus. Ureteres in hac proportione ejusdem erant capacitatis. In universum genus Gaclus longos et capaces habet ureteres; breves vero sunt in Trigla et Cyprino; vix vel non observari possunt in Esoce, cujus renes urinam per fissuram immediatim in vesicam effundunt, quod etiam jam alii observarunt in codem pis- 
pisce et in Anarricha Litpo, qui loco fissurae Esocis duas habet papillas per quas urina in resiçam transit (ibid). Ostium urelerum in vesicam ducens valvula quadam est clausa, neque mirum, nam sine ca facile urina retroiret ad rénes ob situm horizontalem partium. In Trigla haec valvula facile conspicitur, si vesica urinaria aperta est. Etiam liujus valvulac praescntia jam patel ex eo, quod aër et mercurius vesicae inmissi diffacillime in ureteres urgeri possunt, uti in IInuraena. Anguilla accidit, et in Trigla IIirundine; in aliis, l'erca fluviatilis, v. c. valvula illa nulla vi premente cedit, ita ut prius vesicae rupturam quam transitum mercurii in ureteres efficeremus. cum tamen in omnibus mercurius facile ex urcteribus vesicam intrat.

Vasa sanguifera ab intcstinis, ovariis, et cauda uretères adeunt.

VESICA, U RINÄR IA. :

Vesica urinaria ea est systemalis uropoëtici pars, qua urina in renibus secreta relinetur el colligitur, ne scilicet continuo eflluat et ut ibi adhuc partes aquosae absorbeantur. - Gum vero non omnes pisces resica sint instructi, prinum paucis verbis dicam, quomoglo loc systema deficiente vesica constitutum sit.

Nulla certa adest regula, qua a priori dijudicare posscmus piscem aliquem vesica instructum esse vel nou instructum esse. Ureicres si vesica clesideratur solitarii vel sibi invicem appositi descendunt usque prope ad anum et prius vel serius sese conjungunt ut per ostium commune urinam cffundant. Talem dispositionem nobis offert Exocoetus evolans cujus ureteres a renibus ad parietcs abdominis descendunt ot prope ad anum pone intestinum rectum se conjungunt.

Hoc porro ita est in Petromyzonte fuviatili et Cobili fossili, quorum ureteres convergentes descendunt et per parvam papillam in posteriore margine ani urinam emittunt. In Cobili prius ureterés comfluunt quam in Petromysante, quod ex Tabula I. Fig. c. d. videri potest.

Rajae etiam et Squali secundum Clar. Cuvier vesica sunt destituti (No.5. Tom. 4. pag. 640). Interim Relsius, e Squalo Glauco vesicarum quandam speciem non tantum descripsit, verum etiam icone illustravit. Ureteres, duo, inquit, a renis inferiori et auleriori parte in isto sulco initium capiunt, recurrentes sensim crassiores fiunt et in duas oblongas vesicas dilatantur, ex quibus coarctatis oriuntur urcthrae, quae urinam per apicem papillae cloacae emittunt in cloacam. ( $N^{\circ} .26$. pag. 8.)

Ftiam Home in unatomica sua descriptione Squali maximi dicit: "Les uretères se 'terminoient dans une cavité ovale separée en deux par une choison imparfaite, siluée dans les environs de l'anús, et regardée comme pouvant étre l'analogue de la vessic uri-

naire." ( $\mathrm{N}^{\circ}$. 19. pag. 245.) 
In Rajis equidem receptaculum reperi cum renibus communicans et in cloaca se ap. periens; id autem, quin vera vesica sit, nemo dubitabit. Haecce mea observatio etiam probata videtur tabula decima ex opere Monroi a Schneidero edito, quae tabula est duodecima in opere originali. Nam licet etiam Monro vesicam urinariam Rajae uon agnoscat; tamen sua delineatio harum partium, quae Tabula mea II. Fig. 4. proponitur, mihi aliquomodo praesentiam vesicae urinariae probaro videtur. Si enim Tab. mea II. Fig. 4. quae est.ex opere Monroi, consideremus, easdem partes delineatas vidimus, si a differentia sexus abstrahamus, quae in iconibus Tab. I. Fig. 1. 4. et Tab. II. Fig. 5. os: tenduntur.

Ea enim pars Tabulae II. Fig. 4. Lileris KK indicala, cloaca est, quae etiam Tabula 1. Fig. 1 et 2. aperta conspicitur.

Cavitas porro circa D nil est nisi vesica ipsa, resecta pariete superiore, quae eadem vesica Tabula II. sub literu e est delineata, inlegra pariete superiore.

Urcteres in hanc cavitatem finem faciunt, quod patefit stylo D ureteri immisso.

Haece nunc vitiosae delineationis causa facile perspicitur, si in lispositionem et conjunctionem harum partium accurate inquiramus. Cum enim superior pars vesicae urinariae arctissime cohacreat cum pariete cloacae inferiore, salis liquet, horizontali et transversali sectione, qua cloaca est aperta, simul parietem superiorem vesicae esse resectam. Eundem adspectum certo hae partes praebuissent in tabulis meis, si tali transversali sectione cloacam aperuissem, vesica a cloaca, resecando telam cellulosam, nondum separata. Pars denique Tabulae II., Fig. 4. Litera I indicata, quam auctor dicit: pa" pillam, peni similem per quam urina emittitur." nil est nisi urethra, cujus directio Tabula I. Fig. 2. indicatur stylo $g$ ei inmisso, cujusque orificium Tabula 1. Fig. I. proponitur sub litera $e$ :

Vesica urinaria est receptaculum diversac magnitudinis ac capacitatis in posteriore parte cavi abdominalis positum.

In paucis transversim est collocata, uti in Cotto Scorpio (Tabula I. Fig. 4.) et in Rajis (Tabula'I. Fig. 1, 2. et II. Fig. 5.); in universum diameter longitudinalis cum eodem totius corporis convenit. (Pleuronectes, Perca, Cyprinus, $\boldsymbol{E}$ sax et alii).

Supra et pone intestinum rectum vesica locum occupal; inferius juxta eam partes genitales internae reperiuntur. $\mathrm{Ab}$ hacce regula unica aberratio mihi est cognita in Pleuronecte Solea, cujus posterior vesicae pars extra cavum abdominale esi locata, eo scilicet loco quo in caeteris sui generis ovaria collocata observantur. (Tabula II. Fig. 2.)

Forma 'vesicae in' quovis fere genere diversa cernitur. Sic ovalis reperitur (Zoarces, Cyprinus (Tabula II. Fig. 3.) Trigla (Tabula I. Fig. 5a)) vel magis cylindrica finibus rolundatis (Esox Lucius, Perca); bilobata id est, ureteres ad fundum sunt inserti 
et loco huius vesicae duo adsunt sacci conici diversae magnitudinis, ad urethram con venientes. (Gadus Callarias (Tabula II. Fig. 2a) Raja (Tab.: II. Fig. 5.) In sprram est contorta vesica urinaria in Clipea Harengo; diversis cornubus est praedita in Gadorum genere. Cernitur porro sub forma farciminis (Pleuronectes Tab. II. Fig. 2c. 2g.) vel refert sacci figuram (Anarrhichas Lupus ( $\mathrm{N}^{\circ}$.2. pag. 171.)) est pyriformis etc.

Ureteres pro diverso genere diversa ratione vesicae sunt inserti. - Sese effundunt :

a.) in fundum vesicae, quod saepe observatur (Cyprinus', Tab. II. Fig. 3. Gadus Tab. II. Fig. 2a, 26. 2f: Pleuronectes ibidem Fig. 2c. [ex hoc genere excipiatur Plenronectes Solea]).

b.) in corpus vesicae (Zoarces, Pleuronectes Solea (Tab. II. Fig. 29.)

c.) in collum vesicae (Anarrhichas Lupus.)

d.) in collum et simul in corpus vesicae (Gasterosteus Spinachia Tab. 11. Fig. 2co) Duo enim ureteres a superiore parte renum urinam in corpus vesicae ducunt, deinde ex inferiore parte quatuor ad quinque ductus partim ad collum tendunt partim ad corpus resicae.

e.) in fundum et corpus vesicae (Raja Tabula II. Fig. 5.) duo ureteres urinam ex anteriore parte renum in fundum vesicae ducunt, id est in partem urethrae oppositam; caeteri ureteres in corpus vesicae infundunt urinam.

Nunquam ureteres in cornua fimem faciunt.

Vesiea urinaria piscium ex duabus membranis confecta videtur, interna scilicet quae tenuior, magis mucosam et externa fortior, fibrosam referens uaturam.

Fibrae musculares, in vesica conspiciuntur longitudinalẹs et circulares, quarum vì musculari fit, ut in pisce mortuo saepe valde parva et contracta reperiatur. Sic in Esace Lucio, vesica contracta erat ad 38 metra, dum aëre inflato usque ad 55 metra volumine augebatur.

In loco quem occupat, vesica retinetur primo ope ureterum et urethrac; porro proprio ligamento, quod adest inter vesicam et ovaria vel testes; denique etiam peritonaeum et tela cellulosa qua cum. abientibus partibus est conjuncta, multum conferunt ad locum suum servandum; in nonnullis enim generibus (Pleuronectes) tola vesica, in aliis pars tantum vesicae a peritonaco tegitur ac in Gadorum genere.

Iu nonnullorum piscium vesica semper urina reperitur, (Zoarces vrviparus) $\left(N^{\circ} .17.\right)$ : in aliorum hoc numquam obtinet (Cyprinus). Etiam in Pleuronectibus saepe urinam reperi et in Anguilla Muraenca imo calculum urinarium pisi magnitudinem referens; figurae irregularis, coloris ex flavo albi.

Cornua nonnullorum generum vesisae propria, sunt appendices, cum pyloricis intestinorum comparandae, si nempe de figura sermo est. In is nunquam urina reperitur.

C 2 Cor. 
A. J. D. STEENSTRA TOUSSAINT;

Cornua non possident Pleuronectes ". Cyprinus, Esox, Frigla, Perca, Muraena. Ex genere Gadorum aliae species cornua habent aliae iis carent. Sic Gadus Molva, Pollachius, Aeglefinus et Callarias magna habent cornua (Tab. II.). Gadus vero Merlangus vesicam habet sacciformem.

Cornua etiam reperiuntur in genere Cotto et Agono. Magnitudo vesicae relativa ad corpus vel ad partes vesicam ambientes indicari nequit, cum nulla certa ratio vesicam inter et corpus vel ceteras partes observatur, ut vero aliquomodo perspiciatur, quaẹım sit magnitudo harum partium, tabulam hic addam mensurae ureterum, resicae et cornuum in diversis generibus.

Omnes partes erant recentes et aëre inflato extensae.

$$
\text { T A }
$$

mensurae vesicac, ureterum et cornuum.

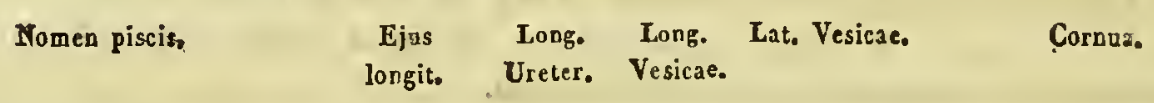

Gadus Merlangus

Gadus Callarias

Gadus Callarias

Esox Lucius

Trigla Gurnardus

Cyclopterus Lumpus

Pleuronectes. Platessa

Pleuronect. Borealis (Fab.)

Zoarces viviparus (Cur.)

Gadus Molva
$10 \frac{1}{2}$ dig. $\quad \frac{3}{4}$ dig. 2 dig. $\quad \frac{1}{4}$ dig.

$11 \frac{x}{4}$ dig. $\quad \frac{x}{2}$ dig. $\left\{\begin{array}{l}\frac{5}{T^{2}} \\ \frac{1}{4}\end{array}\right.$

3 ped. $1 \frac{3}{4}$ dig. $\left\{\begin{array}{l}\frac{5}{5} \\ \frac{\pi}{2}\end{array}\right.$

$16 \mathrm{dig}$.

2 dig. $\frac{x}{3}$ dig.

$14 \frac{1}{2}$ dig. $\frac{x}{12}$ dig. $\frac{1}{2}$ dig. $\frac{1}{3}$ dig.

$7 \frac{\mathrm{T}}{2}$ dig. $\frac{\mathrm{T}}{3}-\frac{\mathrm{T}}{2}$ dig. 1 dig. $\frac{3}{4} \mathrm{dig}$.

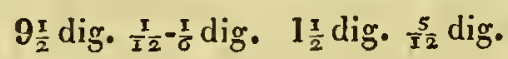

20 dig. $\quad \frac{1}{2}$ dig. 3 dig. $\frac{1}{2}-\frac{2}{3}$ dig.

10 dig. vix $\frac{\pi}{2}$ lin. 1 dig. $\frac{x}{2}$ dig.

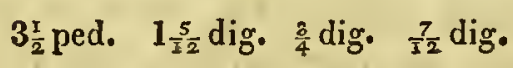

nulla

(1 dig. long. $-\frac{x}{3}$ d. late ( $\frac{5}{6} \mathrm{dig}$. long. $-\frac{1}{3}$ lat.

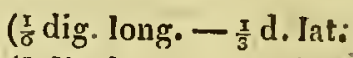
$\left(\frac{\pi}{2}\right.$ dig. long, $-\frac{\pi}{3} d_{\text {. lat. }}$
$\frac{\mathrm{I}}{2}$ dig. long. $-\frac{5}{12} \mathrm{~d}$. Iat. $\frac{1}{2}$ dig. long. $-\frac{1}{5}$ d. lat. $\left(N^{\circ} .17.\right)$ 
D R E T II R A.

Urethra ultima systematis uropoëtici pars est ductus vel meatus angustus a vesica ortus, per quem urina foras expellitur. Finis externus in cutem transit. Nulla vesica urinam immediatim foras eliminare potest, sed semper adesse debet instrumentum vel organon quoddam urinam duccus; idque, nulla ratione formae habita, urethram appellare licet.

In plurimis piscibus externus urethrae adspectus nil nisi parvam offert fossam, ex qua pressis partibus vicinis papilla prominet parva ostio minimo instructa. Aër si per hoc ostium inflatur, resica et ureteres extenduntur. Haec constructio si locum habet, vulgo ductus excernentes ita sunt collocati ut anteriora versus primo conspiciatur anus, cui im. mediatim adjacet ostium ductuum ovariorum vel seminalium; itaque ut denique reperiatur urethra, per septum qroddam distincte separata a prioribus. Id ita observatur in Trigla Hirundine, Cotto Scorpio, et Cataphracto et multis aliis ( $\mathbb{N}^{\circ}$. 17.).

In Rajis urethra hiat inter ostia ovariorum vel seminalium ductuum in cloaca, ubi étiam ostium intestini recti reperitur. Tabula I. Fig. 1 et 2.

Rarius haec papilla urethralis ex ano prominet; sunt autem nonnulli quorum papilla longitudine nonnullarum linearum ita propendet, ut etiam pro organo ad systema sexuale pertinente a nonnullis haberetur. Sic Cuvier dicit de Blennio ( $\mathbf{N}^{2}$. 26. tom. 2. pag. 236.): "Plusieurs sont vivipares, et ils ont tous et dans les deux sexes près de l'anus in tubercule qui paroit leur servir pour l'accouplement." Et etiam Nilsson (N. 29. pag. 91.) dicit de Callionymo: "Monoganiam et Cyclopteri, Gobii et ceteri celebrare videntur, appendice cnim carnosa conica juxta anum instructi sunt, cujus analogia cum pene mammalium in Cycloptero praesertim cernitur." - Etiam Rathke de hac papilla (N.25. pag. 28.) dicit: « Man darf sie als Andeutung einer Ruthe betrachten."

Haec vero sententia ut vera esse possit, ductus ovariorum et seminales sese per hanc papillam aperire oporterent; itaque quod cum locum non habeat, nulla relatio aderse potest papillam inter et systema genitale. - Papilla urethralis semper propendens repcritur in genere Gado, Cyprino, Petromyzonte, porro secundum alios in genere Siluro, Gobio, Zource, Blennio, Callionymo et prae ceteris in Cycloptero Lumpo. Ex his omnibus unicus tantum Gobius secundum observationes mili cognitas ita constructas habet has partes, ut semen et ova per hoc ostium papillae urethralis foras eliminentur. Concedere igitur debemus, esse pisces in quibus ?urina et semen vel ora per unam eandemque aperturam climinantur; non ideo autem hanc papillam membri ge• nitalis munere fungi, cuique palebit. 
Neque verisimilc est per lias papillas coitum locum habere, cum in Gobio mare et fernina ejusdem sint magnitudinis.

In ceteris piscibus haecce papilla solummodo est urethra, et nil nisi urina per eam efluere potest. 'Distincte'vulgo a ceteris ductubus excernentibus separatur septo intermedio. Etiam in Cycloptero Lumpo cujus papilla urethralis unam et dimidiam ad duas lineas longa est, urinae excretione' tantum adaequat. Foramen enim sub litera $a$ ( $\mathrm{Ta}$ bula II. Fig. $2 d_{\text {. }}$ ) est ostium ovariorum; hinc papilla magno foret impedimento, si coitum efficere deberent Cyclopteri. Magis etiam illam papillam urethram esse constat! si pisces contemplemur, quorum systema uropoëticum prorsus a systemate genitali et a tractu intestinali separatum est. Talem enim dispositionem partium jam ante me alii observarunt apud omncs: Pleuronectes; excepto Pleuronecte IIippoglosso, qui eas partes ac in Perca fluviatili dispositas habet.

- In ceteris vero Pleuronectibus anus tantum et ostinm ovariorum vel seminalium due turum in margine corporis suát collocati, dum papilla rnbra urethralis plures saepe lineas ab his remota in latere oculorum reperitur.

De fabrica urethrae ob tenuitatem partium in plurimis nostris piscibus difficile est, certi aliquid pronuntiare.

Delineavi Tabula I. Fig. 3.' urethram Rajae Rubi longitudinali sectione apertam. Fuit imprimis confecta ex compacta tela cellulosa, et in interna facie perspicue apparebant fibrae longitudinales ad lineam rectam concurrentes. Facies interna membrana tecta erat naturae mucosae: Similem constructionem etiam in ceteris piscibus suspicarem: Vasa sanguifcra $a b$ intestinis et vesica urethram adeunt. 


\section{$\mathbf{P}$ A $\mathbf{R}$ S II.}

\section{COMPARATIO PHYSIOLOGICA ORGANORUM URINAM SECERMENTIUII CUN IISDEM PARTIBUS RELIQUORUM ANIMALIUM.}

Functio illa, quam secrelionem nuncupamus est actio, quae omnibus corporibus vi vitali praeditis, est propria, quaeque aeque magni est momenti ad! individui quam speciei conservationem. Secretionum omnium fons est sanguis; attamen non est credendum. humores secretos jam in sanguine contineri; e contra statuendum 'videtur 'elementa quidem humorum in sanguine adesse, sed novas et diversas inire combinationes in diversis organis, ut varii humores ita parentur. - Nihil in physiologicis. liac functione est ob-. scurius, cum non tantam observamus differentiam organorum quam pro diversa natura secretionum expectare licct, et cum omnino in minimis viscerum elementis perágantur, quorum fabricam difficulter cognoscimus. Etian de ratione qua liumores secreti e san. guine perveniant in ductus excretorios multae prolatae sunt theoriae, quarum duae imprimis Ruischii scilicet et MIalpighii, memoratu sunt dignae. Horum ille enim docuit, vasa semper subtiliora transire in ductus excretorios, sine materia interposita. Malpighius vero statuit, ductus excretorios finibus clausis et.vasis perreptatis terminari, quod si verum, sequitur humores ex vasis sanguiferis transsudatione organica in cluctus excretorios transfundi. Quae ultima Malpighii sententia vạriis probalur observationibus a pluribus viris doctis factis. Sic Weber vidit in avium salivalibus glandulis mercurio repletis ductus excretorios terminari clansis et rotundis vesiculis. Ifust ejusmodi vesiculas replevil ad extremos tubulos uriniferos Ranae viridis el refert las vesiculas jam ipso oculo posse distingui, porro etiam avium tubulos uriniferos cavos clausosque terminari. Observationibus deinde Mulleri de his cellulis in ovium embryonibus, clarissimi Rathke de ductubus parotidis et multorum aliorum, Malpighii sententia praevalere videtur.

Inter multivarias 'secretiones, 'quae in corpore animalium perficiantur, secretio urinae certe magni est momenti et longe plurimis communis. Non enim vertebrata solummodo secernunt urinam, sed etiam insecta ét mollusca humorem eliminant, quem pro urina plures salutarunt. ( $\mathrm{N}^{\circ} .10$. et $\mathrm{N}^{\circ} .15$ ) In animalium classibus quatuor superioribus, in rertebratis scilicet, urinae princeps organon esse renes, demonstratur ex similitudine humoris in iis reperti in morbis in quibus cum ureterum destructione vel obstructione 
renes urina distenti reperiuntur, ut et ex cessante secretione, quando renes calculis obstructi sunt vel cultro excisi.

In inferioribus animalium classibus alius adesse debet fons, cum renes deficiant; sic Clar. Meckel vasa ita dicta Malpighiana insectorum, urinam secernentia dicit (No. 10.) et doctissimus Wilbrand,perhibet urinam in Gasteroporlis secerni in sacco purpureo, in Cephalopodis in resica atramentaria.

De genesi vel primaria formatione rcnum in diversis animalibus Clar. Rathke in: primis observationes fecit: "Auch bei den Schlangen, inquit, erscheinen während des Fruchtlebens, gleicherweise wie bei den Vögehn und Säugethieren zwei auf beide Seiten. Hälften des Körpers vertheilte Gebilde (Wolfsclıe Körper) aus denen nieht blos die inneren Geschlechtswerkzeuge, sondern; auch die Nieren zu entstehen. scheinen, und die sich einige Zeit nach ihrem Auftreten theils durch ihre Grösse, theils auch durch ihre Röthe, vor allen übrigen Eingeweiden auszeichnen. - Da sie bei, den Sclilangen und allen nach-höher siehenden Wirbelthieren, wie sich weiterhin überzeugend ergeben wird, in ihrem Baue eine grosse Aehulichkeit mit den Nieren der Fische und vielẹr Amphibien besitzen, so werde ich sie fortan um für sie bei allen damit versehenen Thieren einen gemeinschaftlichen Namen zu haben, die falschen Nieren nennen.", (№.22. Tom. 1. pag: 23.)

Organa haec mox indicata in serpentibus $v$, c. deseribit, tamquam viscera in longitudinem extensa, angusta et tenuia.

Forma uniuscujusque in primo dimidio vitae foetalis est cylindri in duas partes divisi. Post aliquod temporis spatium hoc organon latitudine magis, quam quidern crassitie incressit, anterius paululum attenuatur in apicem rotundatum fuem faciens. (ibidem pag. 33.)

Pagina 35 inquit : renes spurii et ureteres spurii sensim evanescunt, postquam animalia in lucem sint edita, ut ne vestigia quidem horum organorum supersint.

In mammalibus sequenti modo haec organa describit: renes spurii sese extendunt per totum cavum abdominale, anterius pericardium attingentes. Formam exhibent spiralem crassitiei modicae, ratione habita ad longitudinem. Non solum dorso proxime adjacent sed intime cum eo concreti sunt. In externo margine hujus organi, magna decurrit vena, multos ramos ex superficie et intimo organo suscipiens, quae postquam venam jugularem sui lateris receperit sese conjungit cum eadem vena lateris oppositi et in cor se effundit. (ibidem pag. 47.)

Ex quibus nunc organis oriri testes et ovaria Cl. Rathlie certum, dicit; renes, vero ex iis formari ipse dubitat. De renum formatione enim in serpentibus, disputans (ibid. pag. 28. \$. 5.)

ฯ Die eigentlichen. Nieren, inquit, enstehen später, als die falschen, Nieren, viclleicht

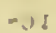


sogar später, als die Geschlechts-Theilc. Sie kommen zum Vorschein oberlualb der falsehen Nieren, und liegen daun nahe der Kloake zwischen jenen Eingeweiden und der Rückenwand der Bauchhöhle. Woher sie aber ihren Ursprung nchmen, lianu ic:h nicht mit Gewissheit angeben: Vielleicht wachsen sie aus den falschen Nieren horvor : wenigstens sind sie mit diesen anfänglich fest verklebt."

Quam vero hypothesin de genesi renum ex renibus ita dictis spuriis non omnes viri docti amplectuntur. Jacobson enim ( $\mathrm{N}^{\circ}$.39.) renes extra peritonaeum locatos esse contendit, dum rencs spurii in propriis plicis vel saccis locum occupant. Etiam. Clar. MI üller in suo opere ( $\mathrm{N}^{\circ}$. 38.) hanc sententiam rejicit.

Deinde Clar. Rathke de renibus ita pergit: postquam apparuerunt ipsi figuram exhibent duarum laminarum tenuium angustarumque, ejusdem fere longitudinis. Aliquan. to post dextra magis longitudine augetur quam sinistra.

Ureteres statim jam ab initio tolos renes ad longitudinem percurrunt el hiant in cloaca prope ad ostium ductus excretorii renum spuriorum (corpora Wolfiana) cum quibus postea ostium habent commune.

De Lacertae crocene et Lacertae agilis renibus sequentia habet: (ibidem pag. 39. §. 13.) renes ipsi, inquit, etiam originem habere videntur ex corporibus Wolfianis. Apparent renes, dum dicta corpora se etiam extendunt usque ad cloacam, in ejus facie superiore, et sensim sensimque partim anteriora versus, partim supra claacam increscunt. Si renes Lacertarum cum is in serpentibus comparemus, eo differunt quod numquam a cloaca recedant.

In embryone Crocodili nilotici renes etiam extensi visi sunt usque ad cloacam, anterius usque ad finem renum spuriorum, nulla vestigia loborum exbibentes.

Hi vero lobi exprimebantur fortibus incisionibus in alio Crocodilo, qui jam ante aliquod tempus ovum reliquit, ut igitur loborum formationem serius nasci, credere fas sit.

In Blennio vivipara Rathlie haecce corpora Wolfiana, ex quibus renes mammalium, avium cet. originem habere credil, numquam reperit. ( $\mathrm{N}^{\circ} .22$. Tom, 2. pag. 32 ). Renes vero, inquit, mox jam apparent, paululum prius fortasse quam hepar. Renes primum oculis sese offerunt, tamquam lamina substantiae gelatinosae, rubescentis, dia. phanae. Intime dorso adhacrent, formam habent longam valde angustam et fere per totum cavum abdominale sunt extensi. Posterior pars licet etiam valde tenuis melius conspicitur quam anterior, quae vix oculis percipi potest, ita ut difficillime finis anterior indicari possit. Videtur igitur haec lamina primum formari in posteriore cavi abdominalis parte, ut dein sensim anterius extendatur. Sensim nunc hae laminae mox descriptae in posteriore terlia parte ubi coalucrunt, mole augentur ct jam nonnullos dies antequam ex ovo prodierit animal, duas inlumescentias perhibent satis longas, posterüus 
in apicem excurrentes. Deizceps dé eo ejusdèm piscis incrementi gradu, quo ôum quicom reliquit, sed in lucem nondum est editus, ita de renibus pergit (ibidem pag, 54 ).

Progrediente incremento embryonis eliam renes molc augentur.'Para anterior renum nunc imprimis ciassior fit,- licet numquam 'codem fradu ac posterior pars. 'Jàm ve ro eodem tempore in media lnmina tum 'superius tum jnferius fośs formatỉr, quae cö est profundius, quo magis posteriora petil ; dividit harc fossa rnes aliquombato in 'luos, ita tamen ut per fotam vitam inter se conjuncti maneant: antegnam igitur foetus red linquat matris abdomen rencs 'eandem jam tenent formám et dimerionios quàs próveetiore aetate prodeunt.

Facies inferiol renum peritonseo tecta, laevis cst in cmbryone, sine ullis striis vel sulcis transversis.

In facie superiore vero tales striae licet valde suparfeiales, modicis interrallis inter-

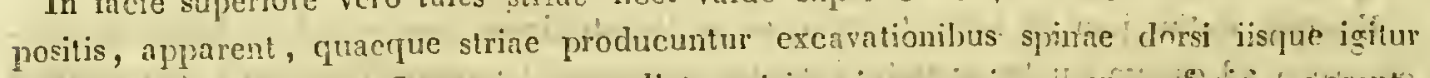
frompte respondent. - Jamantequam dictae striae in superiore renim fà cie apjarent, in intena horum orghopum substantia restigia prima rasolum uriniferorum sè palam fa: cere incjpiunt.

Memoratu etian est dignum rasá ùrinifera primum in posteriore párte rènum formari et omnia simplicia esse, sine ullis ramis (ibidem jag. 55. ).

Progrediente actate cnıbryonis vasa urinifera longitudine valde increscunt el vario modo convolvuntur et flectuntur, ut renos, aucta simul massn interposita, sensim dersiores fiant. Vasa, etiam sanguifera sensim mogis perficiuntur et extenduntur per renum mássam, quo colore rubicundo induuntur.

Vesica urinaria tum denum apparet, si renes notabilem inerementi gradum jam attigerint. Prima ejus vestigia conspiciuntur paulo post ruptum velamentum ovj externum. Forman tunc refert parri noduli, ad finem renum inter: hos et tractum intestinalem locali, et evidenter magis cum renibus quam cum intestinis coliaerct. Cito nunc hóc organon increscil et nutatur in vesicam ovalem, parielibus tenuibus instructam. ìox vesica urinaria repletur linmore aquoso, qui eam valde extensam tenet, usque dum embryo ex abdomine matris expellatur.

In ruminantibus prima renum vestigia vidit Clar. Rathke in embryone Bovis Tauri longitudine $6 \frac{\mathrm{T}}{3}$ lineae. Parva crant corpuscula in posteriore parte cavi abdominalis, renibus spuriis tecta. Ureterum ne indicium quidem aderat, quos etiam Clar. Hueck et Doct. Gaimard et Gerardin non obserrare potuisse confitentur. In hoc porro embryone renes facile a renibus spuriis separari poterant, ut igitur cum his concretos non esse manifestum esset. In aliis embryonibus, qui a tate paululum erant provectiores, ureteres figuram exprimebant fili semidiapliani a fine anteriore ad marginem interum decurrentis, usque ad rudimentum vésicae urinariae ibi observandum.

Ve- 
Veri est simile, inquit Clar. Rallike (ibidem pag 99.) ureteres e renibus versus ve. sicam explicari; quod etian ob analogiam suspicari licet, cum certum sit in Blennio viviparo v. c. ductus excretorios ovarii et testium ex is progredi.

Ex lis omnibus de genesi renum ex variis aucloribus collectis, patel:

1. Corpora Wolfiana non in omnibus animalibus offendi, in quibus renes adsunt.

2? Renes licet saepe cum corporibus illis concreti sint, tawen saepe sine laesione facile ab iis solvi posse.

3 . Renes semper inime adhaerere ficiei dorsi internae.

Qune cum rera sint, Clar. Mulleri et Jacobsonï sententia, renes ex corporibus Wol. fi:uis non formari, maxime probanda mihi videtur, ut credas, renes formari a vasis sangुniferis, in pariete dorsali cari abdominalis decurrentibus. - Systema uropoëticum iu varïs animalium classibus esse diversum, jum dixi; nunc vero paucis considercmus fabricam hujus systematis in classibus singulis.

Prima vestigia systematis uropoëtici adesse videntur in Insectis et Molluscis, Brugonalelli enim in excrementis Phalangis Bombycis Mori reperit uratem ammoniae ( N $\mathrm{N}^{2}$ 40. tom, 8. pag. 42.), cujus substantiae fons, testibus Rerold et Rengrer, vasa ea sunt cum tractu intestinali communicantia, quibus ab aliis bilis sccernendae oficium - est tributum

Chemica lujus materiae analysis a doct. Wur zer instiłuta ( $\mathbb{N}^{\circ}$. 32.) etiam confirmat - observationes mox indicatas. Vie sogenannten Gallengefässe, inquit, sind demnacli (woll Nierenausführungsgânge ohne drüsigen Bau, und die sogeunnnte Gulle IIarn, wofür auch die Insertion der Gallengefässe an einer Stelle des Darmkanals spricht, wo die Kothbildung. schon im vollen.Gange ist.

Scenndum Treviranum (Isis. H. 157. (IX. 1317.) S. 1253.) liaec memorata vasa in Arena atroce hiant in intestinun recum, ut igitur eorum humor nil ad chylificalionem facere possit.

In Molluseis: Jacobson ( $\mathrm{N}^{\circ}$. 34.) acidum uricum, reperil in sacculo calcareo vel glanula testacea Swammerdamii, Polii, Blumenbaclii et aliorum, et serie experimentorum imprimis de Helice nemorali et Planorbe cornea institutorum luculenter demonstravit acidum uricum huic humori inesse.

G. R. Treviranus eliam in sacculo calcareo ILelicum acidum uricum se reperisse testatur.' (Zeitschrift für Physiologie B. 1. s. 52.) In animalibus vertebris instructis systematis uropoëtici fạbrica ita magis elaboratur et composita magis redditır, ut a piscibus ad mammalia progredientes digito guasi incrementum indicare possimus. Quo inferior sit classis eo majores sunt renes, ratione habita corporis; cum enim in inferioribus minus sit perfectum systema respiratorium, alia via sanguis depuretur necesse est;

D 2

qua 
qua ex causa etiam in his animalibus hepar imprimis et renes quoad volumen valde evolutos conspicinus.

In piscium renibus, uti patet ex anatomica eorum descriptione, nihil fere observatur de penitiore structura quam hominis renes ex gr. prodeunt.

In Amplibiis renes magis quidem ac in piscibus diso constituunt organa separata, scd jam sunt minores. In plurimis piscibus renes uti vidimus totum fere cavum abdominis percurrunt; contra in amplibiis dimidiam vel tertiam partem hujus cavi vulgo occupant, ( $\mathrm{N}$. 21. pag. 653.) attamen in his magis etiam lobati apparent, quam in priore classe.

Avinm renes majores quam mammalium ex plurilus lobulis componumtur et in cavitate propria sunt locati inter corpora vertebrarum lumborim. Renum structura horum animalium ut et piscium, substantiae corticali manmalium renum est similis, sine ullis pipillis vel calycibus, quod jam Ferrein demonstravit. ( $\mathbb{N}^{3}$. 35.)

In ultima nunc et perfectissima animalium classe scilicet in mammalibus, renes maxime perfectos offendimus. Fignura in nonuullis tantum racemnsa est, in aliis sine manifesta in lobulos divisione certe in aclulto statu; constant autem in mammalibus renes ex duabus substantiis, colore et textura diversis.

Substantia extema corticalis est rubicunda, mollis et constat ex permultis vasis sanguiferis vario modo inter se decurrentibus et tenuissima tela cellulosa conjunclis, constat porro ex parvis glomerulis et ex finibus ductuum uriniferorum. Non solum haec substantia externum circuitum renum occupat, sed etiam interius in substantiam medullárem penctrat, ita ut eam ab omni parte circumdet.

Substantia medullaris sive tubulosa, interius collocata, densior est et minus colorata quam corticalis substantia. Constat pro maxima parte ex parvis duclibus membranosis, quibus urina continctur, tubulis urinifcris Bellinianis dictis. Tubuli hi ad fasciculos juneti, convergentes ad hilum renalem decurrunt et formant fasciculos pyramidales Ferreinii, quorum bases ad substantiam córlicalem, apices vero verstis hilum renalem spectant. Apices fasciculorum pyramillalium sub forma papillarum parvarum (papillac renales) prominent; earum numerantur quinque ad undecim. Interdum eliam duae vel-tres pyramides in unam papillam se conjungunt, unde tunc minor papillarum quam pyramidum numerus.

Arteria ad singulum rencm ex aorta venit fere sub angulo recto. Si in substantiam renum intraverit arteria, mulos párvos format ramos inter papillas et pyramides decurrentes, qui eo loco quo finis est substantiae medullaris et corticalis anastomoses formant. Fx arcubús denique', quae ita formantur multi tenuissimi ramuli proveniunt, qui pyramiles jenales circumdantes partim radiatim tendunt ad renum sujerficiem, 
partim in glomerulis finem facimnt. Ex siugulis his glomerulis qui convolutis vasis pitpillaribus constitumeur ductus uriniferi Belliniani ortum ducunt et sensim ad parvos ramos se conjungunt, versus ip pices papillarum renaliım decurrentes.

Ureteres in omnibus animalibus, quibus renes dati sunt reperiuntur. In piscibus vel in vesicam aheunt rel immediatim foras educunt urinam, nti hoc antea jam vidimus.

In amphibiis semper ureteres ad cloacam decurrunt, licet saepe acisit vesica infra cloacam posita, in quam urina ex cloaca recipilur. Reperitur vesica in Ranis, Salamandris, Testudinibus, in nonnullis Lacertis ect. Crocodili contra, plures Lacertae et Scrpentes ea resica carent $\left(\mathrm{N}^{\circ}\right.$. 43.). Liumorem hisce vesicis contentum veram esse urinam testantur Lassaigne et Boissel $\left(\mathrm{N}^{\circ}\right.$. 44.) qui in vesica testudinis acidum uricum observarunt, imo Vauquelin in ca calculum urinarium reperit ( $N^{\circ}, 45 . T o m .1$. pag. 434.).

Ureteres avium urinam ducunt in cloacam, ut cum faecihus misceatur. Attamen Struthio Camelus et Casuarius Indicus solummodo excipiantur, qui receptaculum habeant pro urina per valvulam a tractu intestinali separatum, quod jam perruult (No. 47. p. 2. pag. 154.) observavil, et Geoffroy de Saint-IIllaire (No.46. pag. 438.) accurate descripsit. - Ureteres ad faciem dorsalem pelvis decurrunt, musculosae sunt naturae et ad marginem intestini recti in cloacam hiant.

Es nunc dictis patet resicam (in quam scilicet ureteres immediatim urinam ducunt) omnibus amplibiis datam non esse, neque aves ea vesica instructae videntur exceptis Struthione et Casuario, in quibus quaedam vesicae species reperta est. In mammalibus vero semper vesica urinaria adest, in quam urina ex renibus per ureteres imınediatim effunditur.

Urina quin inter humores maximae compositos pertineat non dubitatur, dummodo ad multitudinem substantiarum altendamus, quae a recentioribus artis Chemicae peritis viris in urina repertae sunt. Multi Chemici huic fluido operam dederunt et de tempore in tempus nova sunt reperta, quae antea nondum erant visa principia.

Urina cum aqua miscetur in omni proporione. Si vero alcokol additur ex urina dejicituntur acidum uricum, phosphates terrestres et alia salia in alcohole minus solubilia.

Urina humana teste Théncard continet praeter aquám, nreum, mucum vesicae, pancam materiem ánimalem, acidum uricum, et. acidum phosphoricum hujus vero loco ab aliis acidum accticum vel lacticum repertum est. - Doct. Proust alia adhuc notat principia uti sulphur, quod notatur calefactione urmae in vase argenteo, quod inde nigrescit.

Animalium mammalium urina imprimis est diversa in carnivoris et herbiforis. Carnirororum urina fere omnia continet principia ac humana; sic urina Leonum, Felum et Canum, quae inprimis a Vanquecin et Chevreul est investigata. In herbirororum urina $\therefore$

D 3 aci- 
acidi urici copia minor est. Secundum Foureroy urina equi continct carbonatem calcis, magnesiae et sodac, benzoatem sodae, sulphatem potassac, chloratem potassae, ureum, mucum et oleum rubrum quoddam, cui color est adscribendus.

Avium urina eadem continet principia ac carnivororum: majorem vero continet copiam acidi, a quo principio etiam est derivanda materia illa alba pulverulenta, quari offendimus in superficie excrementorum horum animalium. Acidi urici copia est in ratione directa cum natura magis minusve azotica alimentorum, uti Wollaston probavit in avibus carnivoris, ' in Aquila, Vulture et aliis, in quibus quantitas acidi urici et uratis ammoniac nsque ad dimidiam repleat partem. In serpentibus urina secuidum Las: saigne est consistentiac butyraccac, indurescit aëri exposita et constat ex acido uricorum fere puro. .

Urina Lacertarum fere est eadem. John Davy in ea Crocodilis practer acidum uricum, carbonatem et phospluatem calcis reperit.

Idem vir doct. etiam observavit urinam Ranarum exceptionem facere in hac classé an:malium; est enim limpida, transparens, insipida, odoris fere nullius.

In universum jam concludendum omies fere urinae species vel per plurimas continere ireum. In urina hominum et carnivororum imprimis plosphates hospitantur; in herbivoris phospliates desunt. Benzoates et carbonates ejus locum supplent, cum praetcrea vulgo produnt indolem alcalinam et odore et colore gaudent specifico, oleo cuidam volatili adscribendum.

Urina in mammalibus paratur in flexubus serpentinis arteriae renalis, dein penetrat in ductus uriniferos et ad papillas defertur, ex quarum orificiis quasi rlestillat. Causae yuibus in renibus urina paretur, plane ignotae sunt. Systema nerveum hanc secretionem gubernare, patet ex co, quod a quovis animi motu urinae secretio mutetur. Sic terror et nervosi affectus urinam pallidam reddunt. Si corpus languet urina etiam maligna fluit. Quoties in morbis vires restituuntur urina etiam ad naturalem indolem redit.'

In ceteris animalibus, avibus scilicet, amphibiis, piscibus etc. urinam 'secerni pro maxima parte ex venoso sanguine Jacobsonius auctor est. Series cnim experimentorum certiorem eum reddit, exsistere peculiare systema venosum in hisce animalibus, cum vena portarum hepatis comparandum, cui secretionem urinae tribuit.

In piscibus hoc systema egomet etiam vidi et descripsi in anatomica hujus tractatus parte, Clar. Jacabson etiam in molluscis observarit magnum venarum numerum confluere ad saccum ita dictum calcareum.

Quod nunc attinet ad secretionem urinae per venas, multi fuerunt qui omnem talem secretionem negarunt.

Dicunt tales nullibi venam adducere sanguinem cuidam organo ad secretionem neces_ 
sarium. Si vero altendimus ad pulmones, qui sanguinem venosum, ut depuretur, accipiunt per arterias pulmonales, quare non acciperemus, sangunem venosum liepati adduci per venam, arteriasum in modum confectam, ut depuretur ac in pulmonibus a carbonio.

Haecce nunc sententia accepta (nam plurimi certe nostro tempore conveniunt, venam portarum inprimis inservire thilis secretioni ) quid - impedit ne idem in renibus verum habeamus? Oculorum testimonio certiores facti sumus, exsistere in lisce animalibus systema peculiare, antea descriptum, jure merito cum systemate portarum hepatis comparandum; et si porro attendimus ad paucas ct minimas arterias, si cum venis scilicet comparantur, statucre coacti sumus, sanguinem venosum in his animalibus tum bilis lum urinae parationi inservire, et igitur duas momentosissimas secretiones in iis perfici a venis ex venoso sanguine.

Cum vero haematosis pro parte secretionibis perficilur, haec nunc perspecta venarum nova functio plura phaenomena nondum rite explicata luce spargit, ct melius aliquomodo intelligimus, quare avium sanguis licet parvi is sint pulmones, tam intense sit ruber et quare amphibia, quorum pulmones, sacciformes. aeque sunt parvi, non solum vivere possint in aëre corrupto, respirationi nullo modo adaptato, sed imo sine respiratione per longum tempus vivere possint.

Inltum. adhuc restat opers, multumque restabit, nec ulli nato post mille secula praecludetur occasio, aliquid adhiuc adjiciendi.

SE I E C A.

TAEULAE 


\title{
T A
}

\author{
E A R U I Q U E EX P L I C A T I O.
}

\section{T. A B U L A. I.}

Fig. 1. Partes internae Rajae Rubi.

a. b. Cloaca duabus incisionibus in utroque latere aperta. Pars inferior sub litera a est reflexa et tegit vesicam urinariam, aëre extensam. dd. ostia ovariorum. inter quae e. orificium urcthrae. c. tractus intestinalis. ff, ovaria. gg. tubae falloppii. h. oesophagus. ii. ductus ovarii sinistri aëre extensus.

Fig. 2. Eaedem partes ac in prima tabula.

a. pars inferior cloacae, in prima tabula reflexa, $\delta$. interna facies cloacae. $c$. likgatus tractus intestinalis. $d d$. ductus ovariorum. e. vesica urinaria. $f$. finis ure: thrae. $g$. stylus, urethrae directionem indicans. $h h$. ureteres. $k k$, renes.

Fig. 3. Vesica Rajae Rubi magnitudine naturali. Ureterum minores rami non beneerant repleti. Urethra sectione longitudinali aperla, fibras ostendit longitudinales, convergentes.

quam

Fig. 4. Ren Rajae Rubi, magnitudine naturali cum vasis suis, tam arteriosis. venosis.

Figura 5a. Pars posterior renis Triglae cum ejus vesica. Ureteres colore rubro sunt delineati; sinus renosus coeruleo colore cst tinctus. 
Fig. b. Renes cum vesica Cotlo Scorpio.

Fig. c. Ureteres in Petromyzontc fluviatili.

a a a a. pars inferior tractus intestinalis, longitudinali sectione aperta. $b$. ureteres. c. papilla urethralis.

Fig. d. Eaedem partes ac in figura c. ex Cobiti fossiti.

\section{T A $\quad \mathbf{A}^{\prime}$ B 'U $\mathbf{L}^{\prime} \mathbf{A}$ ' II:}

"Fig. 1." Inferior facies renis Triglae Itivundinis; cum sinu venoso, longitudinali incisione aperto, ut in conspectum veniant ostia aperta minorum vasorum quae in sinum sanguinem infundunt. Anterius sinus duos format ramos.

Figura 2a. Vesica urinaria e Gado Callaria.

a. ureteres, ${ }^{i}$. urethra. " cc. vesicae sacciformes.

Fig. 2b. Vesica urinaria e Gado Molva.

$a$ ureteres. b. urethra. cc. cornua.

Fig. 2c. Vesica uriuaria Pleuronectis Platessce.

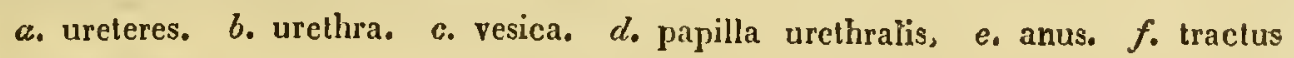
intestinalis.

Fig. 2d. Papilla urethralis Cyclopteri Lumpi.

a. apertura oviductus ad basin urethrae.

Fig. 2e. Systema uropoëticum e Gasterosteo Spinachia.

$a$. vesica urinaria. $b b$. renes. cccccc. diversi ureteres in vesicam effundentes.

Fig. 2f. Vesica urinaria Gadi Aeglefini.

a. ureteres. b. corpus vesicae. ec. cornua. $d$. orificium urethrae. $f$. tractus intestinalis inferior pars aperta.

Fig. 2g. Pars renis et vesica urinaria Pleuronectis Soleae.

$a a$. vesica. $b$, inferior pars renum. $c c$, ureteres. $d$. papilla urethralis.

Fig. 3. Ren Cyprini Rutili.

aa. pars anterior renum ad capitulum incrassata. $b$. locus ubi renes ad unam confluunt massam. cc. magnum vas venosum (vena cava) renes percurrens.

$d d d d$. vasa venosa, quae in lateribus abdominis formata ad renes adscendunt, ut de novo in ramos se extendant. e. vena altera, quae in superiore parte renis formatur. $f f$. ureteres totos rcnes percurrentes. $g$. vesica urinaria cum urethra.

Fig. 4. Est delinatio partium internarum Rajae, quae reperitur in opere A. MIonroi, E 
a Schneidero edito (Vergleichung des Baues und der Physiologie des. Menschen und der Thiere.

A. vertebrae. C. ren sinister, D. seta ureteri sinistro immissa. E. ductus deferens sinister, valde dilatatus in parte inferiore et posteriorc. |I । : ") : , ,

FF. finis horum ductưm, in dextro latere integer; apertus vero in sinistro.

GG. vesica magna, humorem continens profunde viridem, eodemque loco lians

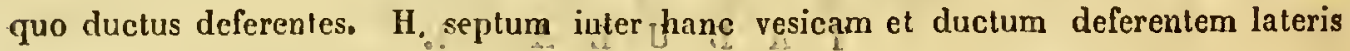
sinistri. I. infundibulum, sive papilla peni similis, per quam urina, semen et contentum vesicarum GG. ducitur ad KK. finis tractus intestinalis sive cloaca communis LL. finis ductuum excretoriorum ex cavo abdominali: M. stylus missus per canalem dextrum.

Fig. 5. Organa uropoëlica ex Raja clavatä.

$a a$. vesica urinaria. b. unethra. c. cloacae superior facies. d. ren dexter. e. columna vertebralis. $f$. membrana renis reflexa. $g g g g g$ ureteres. 


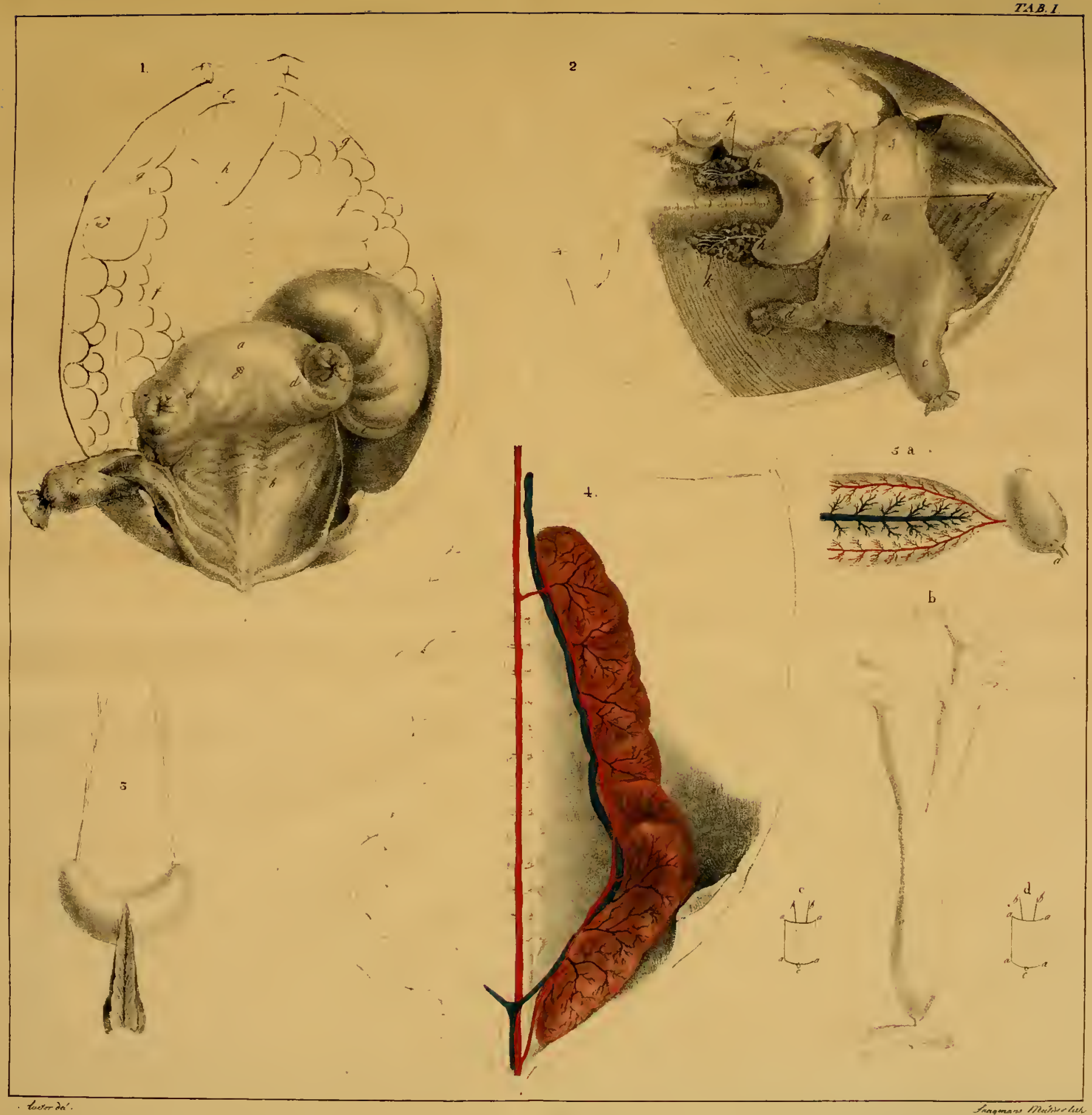





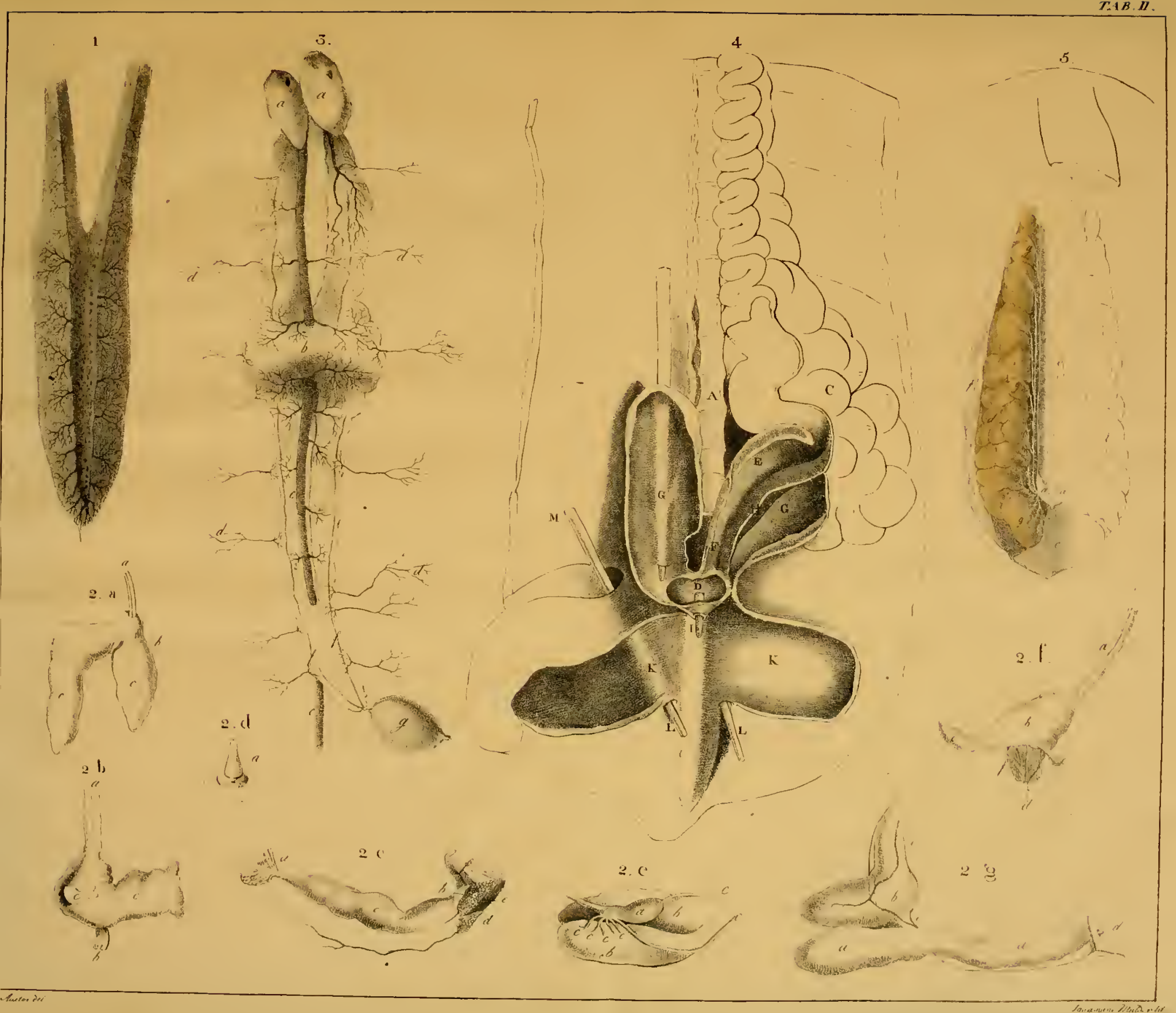






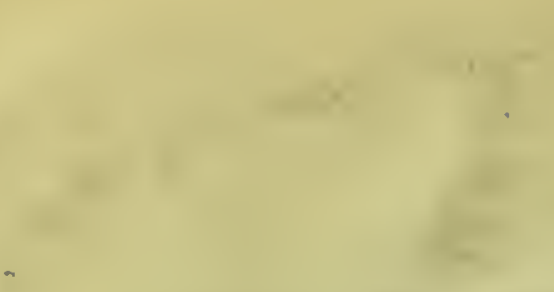

$-$
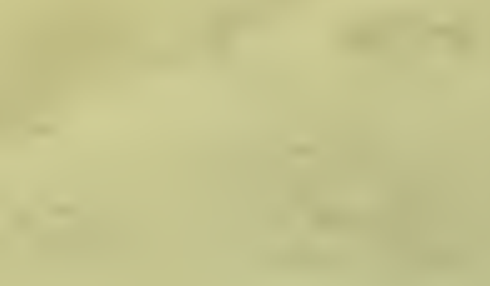

$5=$
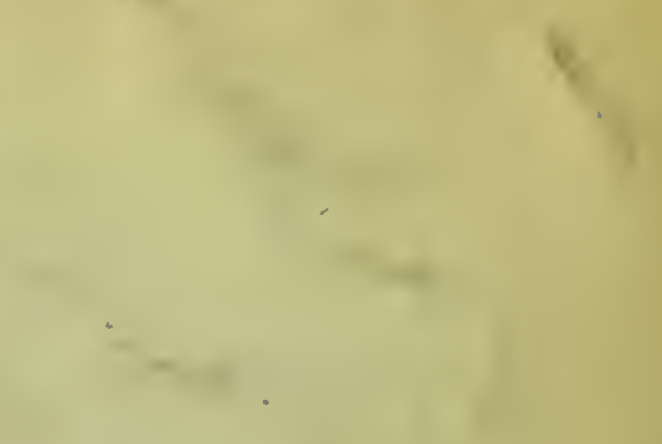

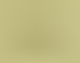

non

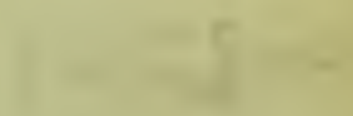

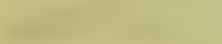

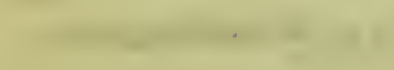

\section{$-$}

$-$

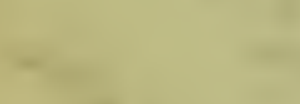

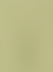

$x^{2}$
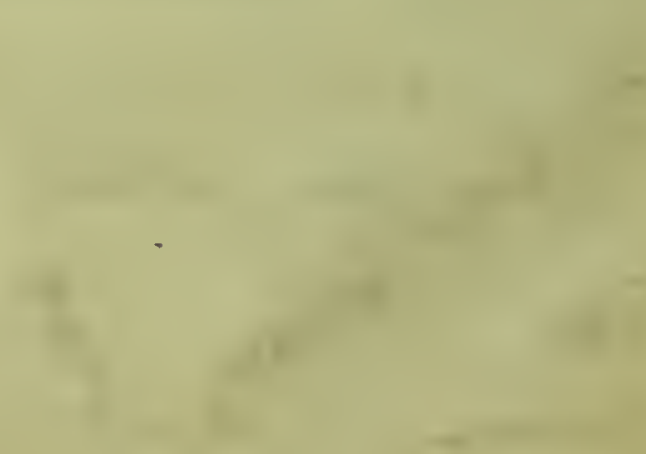

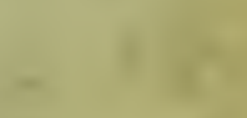

$-$

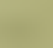

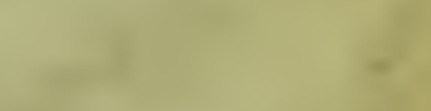

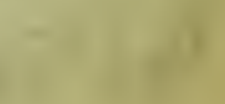






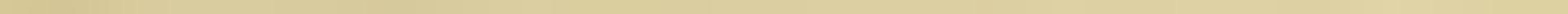




